\title{
Mutually exclusive amino acid residues of L13a are responsible for its ribosomal incorporation and translational silencing leading to resolution of inflammation
}

\author{
RAVINDER KOUR, ANTON A. KOMAR, and BARSANJIT MAZUMDER \\ Center for Gene Regulation in Health and Disease, Department of Biological, Geological and Environmental Sciences, Cleveland State University, \\ Cleveland, Ohio 44115, USA
}

\begin{abstract}
Eukaryotic ribosomal protein L13a is a member of the conserved universal ribosomal uL13 protein family. Structurally, L13a is distinguished from its prokaryotic counterparts by the presence of an $\sim 55$ amino acid-long carboxy-terminal $\alpha$-helical extension. The importance of these evolved residues in the carboxy-terminal extension for mammalian ribosome biogenesis as well as L13a's extraribosomal function in GAIT ( $\gamma$ interferon-activated inhibitor of translation) complex-mediated translation silencing during inflammation is not understood. Here, we present biochemical analyses of L13a mutant variants identifying several mutually exclusive amino acid residues in the eukaryote-specific carboxy-terminal extension of human L13a (Tyr149-Val203) important for ribosomal incorporation and translational silencing. Specifically, we show that mutation of Arg169, Lys170, and Lys171 to Ala abrogate GAIT-mediated translational silencing, but not L13a incorporation into ribosomes. Moreover, we show that the carboxy-terminal helix alone can silence translation of GAIT element-containing mRNAs in vitro. We also show through cellular immunofluorescence experiments that nuclear but not nucleolar localization of $\mathrm{L} 13 \mathrm{a}$ is resistant to extensive amino acid alterations, suggesting that multiple complex nuclear import signals are present within this protein. These studies provide new insights into L13a structure and its ribosomal and extraribosomal functions in model human cells.
\end{abstract}

Keywords: L13a; ribosomal incorporation; nuclear localization; translational silencing; inflammation

\section{INTRODUCTION}

Ribosome biogenesis is a fundamental and highly complex process in all living organisms. In yeast, the biogenesis of a eukaryotic ribosome involves about 80 ribosomal proteins, four rRNAs, 150 assembly factors, and about 70 snoRNAs (Fromont-Racine et al. 2003; Henras et al. 2008). In higher eukaryotes, the pathway is much more complex and the knowledge of the intermediates of ribosome biogenesis is not fully known. This process is energy-intensive and regulated by endogenous cellular inputs as well as mitogenic signals and nutrients (Pelletier et al. 2018). Despite the overall similarity of prokaryotic and eukaryotic ribosomes, their assembly pathways are dramatically different. In eukaryotes, transport of ribosomal proteins from the cytoplasm to the nucleus and then to the nucleolus is a prerequisite for their incorporation into nascent ribosomes (Melese and Xue 1995; Boisvert et al. 2007). Thus, it is presumed that all eukaryotic ribosomal proteins harbor nu-

Corresponding author: b.mazumder@csuohio.edu

Article is online at http://www.rnajournal.org/cgi/doi/10.1261/rna. 071118.119. clear localization signals (NLSs), which are typically relatively short ( 10-20 amino acids), basic sequence motifs. Such NLSs have been predicted, and in some cases experimentally verified, for a number of ribosomal proteins (Moreland et al. 1985; Rout et al. 1997; Rosorius et al. 2000). Some of these NLSs lie within the nonglobular extensions of the rRNA-binding domains of ribosomal proteins (Melnikov et al. 2015). Nevertheless, the identity of individual ribosomal proteins comprising specific preribosomal intermediates present in the nucleus and/or nucleolus is not well established. Studies of yeast preribosomal complexes isolated by affinity purification approaches were complicated by nonspecific protein binding and binding of ribosomal proteins to complexes irrelevant to ribosome biogenesis (Milkereit et al. 2003). In addition, for most individual ribosomal proteins of higher eukaryotes, the amino acid residues critical for incorporation into

(C) 2019 Kour et al. This article is distributed exclusively by the RNA Society for the first 12 months after the full-issue publication date (see http://rnajournal.cshlp.org/site/misc/terms.xhtml). After 12 months, it is available under a Creative Commons License (Attribution-NonCommercial 4.0 International), as described at http:// creativecommons.org/licenses/by-nc/4.0/. 
mature ribosomes have not been clearly defined. Recent advances in X-ray crystallography and, especially, cryoelectron microscopy (Cryo-EM), have improved our understanding of the architecture and organization of yeast (Ben-Shem et al. 2011) and human ribosomes (Behrmann et al. 2015; Zhang et al. 2016; Natchiar et al. 2017) and allowed for direct structural comparison of their individual rRNA and protein components to prokaryotic counterparts. This provided evidence that bacterial and eukaryotic ribosomes evolved from a common structural Ribonucleoprotein (RNP) core with the majority of differences being in the outer shell of the ribosome. These differences include extra rRNA (expansion segments), additional ribosomal proteins, and extensions of conserved ribosomal proteins specifically in eukaryotes. Mammalian ribosomal protein L13a belongs to the highly conserved L13 family of proteins (Ben-Shem et al. 2011) and is a component of the 605 ribosomal subunit and the mature $80 \mathrm{~S}$ ribosome. While depletion of the L13a yeast homolog (L16) causes lethality and impairs ribosome biogenesis in yeast Saccharomyces cerevisiae (Ohmayer et al. 2015; EspinarMarchena et al. 2016), depletion of the mammalian L13a in cultured cell models had no effect on rRNA processing, ribosome assembly, or on the primary activity of ribosomes, global/cap-dependent protein synthesis (Chaudhuri et al. 2007). Thus, despite the substantial homology between the yeast L16 and mammalian L13a, the impact of the two proteins on ribosome assembly and function substantially differs. Mammalian L13a was also shown to play a critical role in rRNA methylation and in addition was found to impact minor protein synthesis pathways, such as those relying on the presence of internal ribosome entry site (IRES) elements in cellular mRNAs (Chaudhuri et al. 2007). We also previously discovered an extraribosomal function of this protein: translational silencing of a group of inflammatory proteins in interferon-gamma (IFN- $\gamma$ )-activated myeloid cells (Mazumder et al. 2003; Vyas et al. 2009). Our work showed that in monocytes/macrophages, IFN- $\gamma$-induced phosphorylation of $\mathrm{L} 13 \mathrm{a}$ leads to its release from the $60 \mathrm{~S}$ ribosomal subunit and assembly into a GAIT (gamma-interferon-activated inhibitor of translation) complex. This complex binds to the GAIT sequence elements located in the 3' untranslated region (UTR) of target mRNAs and blocks translation initiation by inhibiting recruitment of the 43S preinitiation complex to the elF4F cap-binding complex (Kapasi et al. 2007). To drive the L13a-mediated translational silencing of a group of chemokine (CC/CXC) and the chemokine receptor (CCR) mRNAs, the diverse RNA GAIT-elements rely on conservation of RNA structure rather than the sequence to potentiate the binding of GAIT complex and thus resolves the inflammation (Basu et al. 2017). This was verified in vivo in murine models with a macrophage-specific knockout of L13a: Lack of this protein resulted in uncontrolled inflammation and more severe disease (Poddar et al. 2013, 2016; Basu et al. 2014). The
GAIT complex contains three proteins in addition to L13a: glutamyl-prolyl-tRNA synthetase (GluProRS), NS1associated protein-1, and glyceraldehyde 3-phosphate dehydrogenase (Sampath et al. 2004). GluProRS is believed to be responsible for GAIT element recognition and binding, but the function of the other three GAIT complex proteins, including $\mathrm{L} 13 \mathrm{a}$, remains largely unknown. To gain insight into this important innate immune control mechanism that is dependent on the ribosomal release of $L 13 a$, and exploit this mechanism for the therapeutic purpose, it is important to define how the protein gets incorporated into ribosomes. This knowledge is important because amino acid residues responsible for $L 13$ a association with a $60 \mathrm{~S}$ ribosomal subunit would be targeted by the mechanism causing the release of this protein from the ribosome. Toward this goal, our previous work identified Arg68 in the amino-terminal globular domain of L13a as essential for rRNA binding and ribosomal incorporation (Das et al. 2013) and defined the 905 preribosome (present in the nucleolus as a precursor to 405 and 605 subunits) as the principle site/particle for L13a incorporation and L13a-mediated methylation of rRNA. However, this study also showed that nucleolar translocation per se is not sufficient for L13a incorporation into the ribosome (Das et al. 2013). In general, the importance of residues in the eukaryotespecific carboxy-terminal extension of mammalian L13a for processes affecting its ribosomal incorporation and function (e.g., nuclear/nucleolar translocation, rRNAbinding, etc.) and for its extraribosomal function in translational silencing has not been established. In yeast, Saccharomyces cerevisiae, however, the eukaryote-specific carboxy-terminal extension of the rpL16/uL13 protein was shown to be important for early pre-rRNA processing and for stabilization of 60S ribosomal precursors, but was not strictly required for rpL16 recruitment into preribosomes (Ohmayer et al. 2015; Espinar-Marchena et al. 2016). Thus, given that the impact of the L16 and L13a on ribosome assembly and function substantially differs between yeast and mammalian systems, it could not be excluded that uL13 eukaryote-specific carboxy-terminal extension may have acquired additional functions in mammals. We approached this problem by generating and characterizing a new set of mammalian $\mathrm{L} 13$ a variants with targeted deletions and mutations primarily in the eukaryote-specific carboxy-terminal extension domain. Biochemical studies combined with cellular microscopy experiments showed that a set of mutually exclusive amino acid residues of $\mathrm{L} 13 \mathrm{a}$ are responsible for its ribosomal incorporation and GAIT-mediated translational silencing. Contrary to expectations, our experiments did not validate NLSs predicted using structural (Melnikov et al. 2015) and/ or computational approaches (Nguyen Ba et al. 2009). In fact, the nuclear translocation of $\mathrm{L} 13 \mathrm{a}$ was unaffected by numerous amino acid substitutions. Interestingly, however, nucleolar translocation of $\mathrm{L} 13 \mathrm{a}$ was less tolerant of 
such changes. We further found that the eukaryotespecific carboxy-terminal helical extension of $\mathrm{L} 13 \mathrm{a}$ is solely responsible for the protein's role in GAIT-mediated translational silencing of proinflammatory mRNAs. Notably, the three carboxy-terminal residues found to be critical for L13a's translational silencing activity (Arg169, Lys170, and Lys171) were dispensable for its ribosomal incorporation. These data provide new insights into the evolution of eukaryotic (mammalian) L13a and, specifically, into the function of its eukaryote-specific carboxy-terminal helical extension.

\section{RESULTS}

\section{The eukaryote-specific carboxy-terminal extension of human L13a harbors a predicted nuclear localization signal and rRNA-binding site}

Human ribosomal protein L13a belongs to a L13 superfamily of the highly conserved ribosomal proteins from the large ribosomal subunit. The sequence (Supplemental Fig. S1a) and structural alignments (Supplemental Fig. S1b) between Escherichia coli L13 and human L13a show a conserved amino-terminal globular domain and a eukaryotic-specific carboxy-terminal domain. The eukaryotic-specific domain ( 55 amino acid residues in length, residues Tyr149 to Val203 in human L13a) forms a long $\alpha$ helix that bends at its carboxy-terminal end and interacts with a long carboxy-terminal helix of human ribosomal protein L14 (Fig. 1A-C). While the evolutionary significance of the mammalian L13a carboxy-terminal extension remains largely unknown, it is logical to hypothesize that this domain could be responsible for some of the functional differences between L13a and its prokaryotic counterparts. For example, in E. coli, L13 is one of the early assembly proteins of the large ribosomal subunit and is essential for ribosome function (Herold and Nierhaus 1987; Tumminia et al. 1994). In contrast, as described in the Introduction, eukaryotic L13a is not essential for the main function of ribosomes (cap-dependent translation) (Chaudhuri et al. 2007), but impacts rRNA methylation, minor/alternative protein synthesis pathways, and, upon release from ribosomes, translational silencing of a specific set of inflammationassociated genes. In addition, the biogenesis of prokaryotic and eukaryotic ribosomes is clearly different since the nucleolus is the main site of ribosome assembly in eukaryotes, while prokaryotes lack subcellular organelles. In particular, for eukaryotic L13a, we previously showed that the protein translocates into the nucleus and accumulates in the nucleolus during its incorporation into the 905 preribosome (Das et al. 2013). It could not be also excluded that this protein and specifically its eukaryote-specific carboxy-terminal domain could have acquired additional functional roles in higher eukaryotes in comparison with yeast. This suggestion is consistent with the data showing a substantial differ- ence in the outcomes of protein depletion in yeast (Ohmayer et al. 2015; Espinar-Marchena et al. 2016) in comparison with the mammalian cells (Chaudhuri et al. 2007). Thus, as a starting point to investigate the function of the eukaryote-specific carboxy-terminal extension of mammalian L13a, we used two web-based programs to search for potential NLSs within its sequence (NLS mapper [http://nls-mapper.iab.keio.ac.jp/cgi-bin/NLS_Mapper_form .cgi] and NLStradamus [http://www.moseslab.csb.utoronto .ca/NLStradamus/]). These programs identified a potential NLS comprised of residues Lys 159 to Lys 188 within the carboxy-terminal extension. We have termed this sequence NLS-2 as an additional NLS was also predicted within the amino-terminal globular domain of the protein (NLS-1, residues Arg84 to Met118) (Fig. 1A). The potential functional relevance of NLS-2 is supported by previous structureguided analysis of the Saccharomyces cerevisiae ribosome, which suggested that an $\alpha$-helical eukaryote-specific carboxy-terminal extension of the L13a protein might serve as a NLS (Melnikov et al. 2015). We also used a program to identify possible RNA binding sites within the L13a carboxy-terminal extension (RNABindR: http://einstein.cs .iastate.edu/RNABindR). This identified a region spanning residues Arg169 to Lys179 (lying within NLS-2) that may be involved in rRNA interactions (Fig. 1A,B). This putative RNA-binding sequence is in addition to one in the amino-terminal domain (residues Lys53 to Ala75; Fig. 1A) that was predicted in earlier work and shown to be critically important for human L13a ribosomal incorporation (Das et al. 2013). Identification of putative NLS and RNAbinding sequences within the eukaryote-specific carboxyterminal extension of $\mathrm{L} 13 \mathrm{a}$ provided a foundation for functional dissection of this domain.

\section{Eukaryote-specific carboxy-terminal extension of L13a harbors residues important for ribosomal incorporation}

We have previously identified Arg68 (located within the amino-terminal [globular] domain of the protein) as one of the amino acid residues critical for L13a binding to the large ribosomal subunit (Das et al. 2013). This residue was predicted to be located at the tip of the protruding loop region (a position occupied by His60 in E. coli L13) contacting rRNA (Supplemental Fig. S1b). Several other residues within L13a globular domain appeared to be also important for L13a ribosomal incorporation (Das et al. 2013). However, the potential importance of the mammalian L13a eukaryote-specific extension region for ribosomal incorporation and rRNA-binding remained unknown. Web-based prediction platforms NLStradamus and RNABindR predict that this region may harbor a NLS spanning Lys159 to Lys188 and an RNA-binding domain spanning residues Arg169 to Lys179 (Fig. 1A). Obviously, both nuclear and, eventually, nucleolar translocation as 
A

Predicted NLS-2
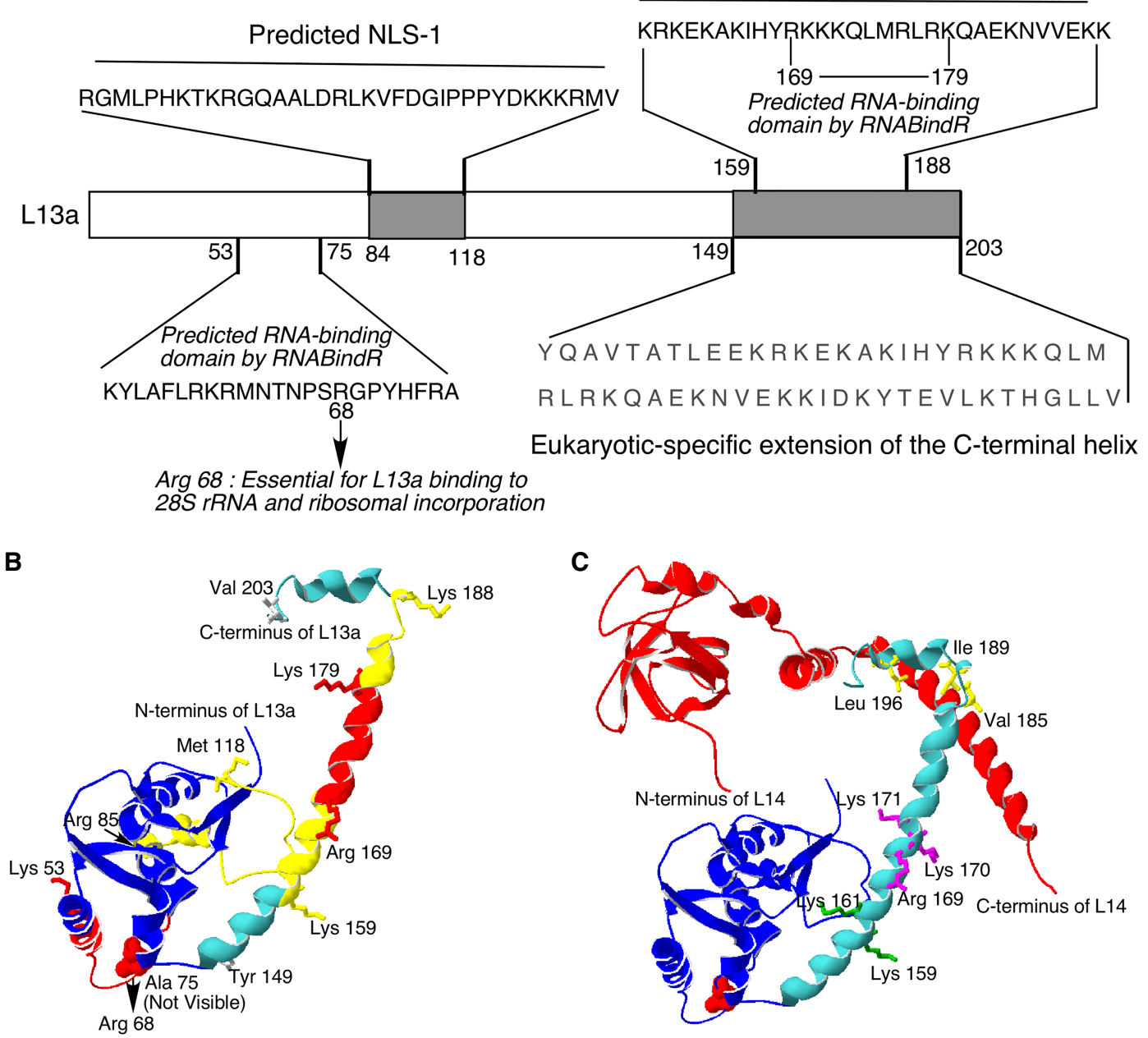

Human L13a structure depicted as ribbon diagram based on CryoEM data

FIGURE 1. Putative NLSs, RNA binding sites, and structure of ribosomal protein L13a. (A) Schematic diagram of the human L13a amino acid sequence with predicted NLSs (Arg84 to Met118 and Lys159 to Lys188) and rRNA-binding sites (Lys53 to Ala75 and Arg169 to Lys179) indicated. Arg68, a residue experimentally verified as essential for rRNA-binding and ribosomal incorporation, is also indicated. The eukaryote-specific L13a carboxy-terminal extension spans residues Trp149 to Val203. (B) Human L13a structure depicted as a ribbon diagram based on the CryoEM structure of the human ribosome at $3.6 \AA$ resolution (Protein Data Bank code 5T2C). The conserved globular core domain is shown in dark blue. Predicted NLSs (Arg84 to Met118 and Lys159 to Lys188) are shown in yellow. Predicted RNA-binding sites (Lys53 to Ala75 and Arg169 to Lys179) are shown in red. The eukaryote-specific carboxy-terminal extension (Tyr149 to Val203) is shown in sky blue. Side chains are shown as sticks for Lys53, Lys159, Lys179, Lys188, Ala75, Arg85, Arg169, Met 118, Tyr149, and Val203. (C) Modeling of the interaction between the carboxy-terminal helices of human L13a and L14 proteins. L13a (blue and sky blue) and L14 (red) protein structures are depicted as ribbon diagrams based on the CryoEM structure of the human ribosome at 3.6 Å resolution (Protein Data Bank code 5T2C). Side chains of the L13a residues experimentally determined to affect L13a ribosomal incorporation (Arg68, Lys159, Lys161, Arg169, Lys170, and Lys171) are shown. The van der Waals radius of Arg68 (also essential for L13a ribosomal incorporation) is shown in red. Side chains of L13a residues Val185, Ile189, and Leu196 interacting with the L14 carboxy-terminal helix are shown in yellow.

well as rRNA-binding abilities would be essential for incorporation of this protein into the ribosome. In addition, observed contacts formed between L13a and L14 carboxy-terminal helices (Fig. 1C) suggest that this region could be also important for L13a ribosomal attachment. Eukaryote-specific polypeptide extensions of the eukaryotic ribosomal proteins frequently appear to interact with each other, resulting in complex networks of ribosomal protein interactions on the outer shell of the ribosome in eukaryotes. It has been generally hypothesized that eukaryote-specific extensions of the conserved ribosomal proteins evolved to accommodate specific, yet largely unknown functions. In order to dissect the function of eukaryote-specific carboxy-terminal extension of the mammalian L13a we have created a set of L13a HA-tagged mutant variants and expressed them in HEK293T cells. Sucrose density gradient centrifugation analysis of individual cell lysates expressing each HA-tagged L13a variant 
coupled with immunoblotting was used to determine the ability of individual variants to get incorporated into the active/translation competent ribosomes (Fig. 2A). First, we have tested, how the deletion of the entire carboxyterminal extension L13a 1-148( $\Delta 149-203)$ affects L13a ribosomal incorporation. We have also expressed this carboxy-terminal extension segment 149-203(41-148) alone. Both L13a fragments failed to incorporate into the 60S, $80 \mathrm{~S}$ ribosomes and polyribosomes and concentrated mostly in the free fractions (Fig. 2A). We have further tested a series of point, as well as double and triple L13a mutants, namely L13a triple mutant Arg169Ala-Lys170AlaLys171Ala, a double mutant Lys179Ala-GIn180Ala and point mutants Lys172Ala, Gln173Ala, Met175Ala, Arg176Ala, Leu177Ala, respectively, harboring mutations within the predicted RNA-binding region (Fig. 2B). Surprisingly, all of these mutants showed no defects in ribosomal incorporation (Fig. 2A). These results suggest that the (predicted) RNA-binding domain within the predicted NLS-2 region of the eukaryote-specific carboxy-terminal extension is not involved in rRNA binding. In contrast, L13a variants with single mutation of Lys159 to Ala or Lys 161 to Ala entirely abrogated the incorporation of L13a protein into 60S ribosomal subunits, $80 \mathrm{~S}$ ribosomes and polyribosomes and were confined to the free fractions (Fig. 2A).

To further test the role of the residues involved in the interaction between L13a and L14 (Fig. 1C) in the ribosomal incorporation of $\mathrm{L} 13 \mathrm{a}$, we have generated the triple mutant Val185Ala-Ile189Ala-Leu196Ala and the double mutant Val185Ala-Ile189Ala. Both of the L13a variants failed to incorporate into the ribosome (Fig. 2A). However, the L13a triple mutant Glu186Ala-Lys187Ala-Lys188Ala (harboring mutations of the residues within the same region, which however do not appear to be directly involved in the interaction with L14) showed no defect in ribosomal incorporation (Fig. 2A). Inputs from the total cell lysates expressing each L13a variant before ribosome fractionations are presented in Supplemental Figure S2. Together, these findings suggest the essential role of Lys159 and Lys161 present within the predicted NLS-2 and of the stretch of residues directly involved in the interaction between L13a and L14 in the process of L13a ribosomal incorporation.

The ribosomal incorporation assay we have used is based on cosedimentation of the expressed Ha-tagged protein with different ribosomal subunits and polyribosomes. To further directly test the in vivo association of the L13a protein variants with 28S rRNA we have used RNA-immunoprecipitation. As such, we have immunoprecipitated L13a-bound 28S rRNA (using anti-HA antibodies) from the HEK293T cells transfected with different HAtagged L13a variants. The presence of 28S rRNA was tested by RT-PCR using primers specific for $28 \mathrm{~S}$ rRNA. Following this approach we tested eight different L13a var- iants, e.g., ribosome incorporation defective Lys159AlaArg160Ala-Lys161Ala, Val185Ala-Ile189Ala-Leu196Ala, (1-148) $\Delta 149-203,(149-203) \Delta 1-148$ and previously made Arg68Ala (Das et al. 2013); incorporation competent Arg169Ala-Lys170Ala-Lys171Ala and Ile166A-His167 Ala-Tyr168Ala and a single variant without predicted NLS-1 and NLS-2 (Fig. 1A) (1-84) $\Delta$ 85-118+(119-148) $\Delta 149-203$. Consistent with the results from our study of ribosomal incorporation through cosedimentation experiments (Fig. 2A), we had not detected 28S rRNA in the immunoprecipitates of Lys159Ala-Arg160Ala-Lys161Ala, Val185Ala-Ile189Ala-Leu196Ala, (1-148)4149-203, (149203) $\Delta 1-148$, Arg68Ala and of the variant without NLS-1 and NLS-2 i.e., $(1-84) \Delta 85-118+(119-148) \Delta 149-203$ (Fig. 3). In contrast, in the same experiment association of $28 \mathrm{~S}$ rRNA with the WT L13a and the incorporation competent (by cosedimentation) mutants Arg169AlaLys170Ala-Lys161Ala and Ile166Ala-His167Ala-Tyr168A was observed. Immunoblotting analysis with anti-HA of the same immunoprecipitates confirmed the equal efficiency of immunoprecipitation for different L13a variants (Fig. 3). The specificity of the $28 \mathrm{~S}$ rRNA immunoprecipitation was confirmed by the absence of the PCR product from $28 \mathrm{~S}$ rRNA obtained using HA-tagged WT L13atransfected cells and beads without coupling with the antibody (Fig. 3 upper first lane).

\section{Mutations in the predicted NLS within the eukaryote- specific carboxy-terminal extension of L13a affect nucleolar, but not nuclear, translocation of the protein}

In order to understand the mechanistic basis of L13a assembly into the mature ribosome, it is essential to dissect steps and mechanisms guiding the subcellular trafficking of this protein and its binding to rRNA. In eukaryotes, most of the events for ribosome assembly take place inside the nucleolus (Henras et al. 2008; Pelletier et al. 2018). Therefore, we have further tested whether defects in ribosomal incorporation (Figs. 2, 3) observed for various L13a mutants could be due to their inability to translocate into the nucleus and/or the nucleolus. Translocation of the L13a variants into the nucleus and/or nucleolus was monitored by the cellular immunofluorescence analysis. Ribosome incorporation-defective and competent HAtagged L13a mutants along with the WT protein were used to transfect HEK 293T cells followed by detection of the HA-tag with Alexa Fluor-488 (green) and nucleolin (a marker of nucleolus) with Alexa Fluor-594 (red) within the DAPI stained nuclei. As expected, this experiment showed that WT L13a is able to completely translocate into the nucleolus without any nuclear retention (Fig. 4). Interestingly, all of the ribosomal incorporation-defective as well as predicted NLS-deleted mutants, for example, 1-148 (4149-203, with eukaryote-specific carboxy- 
A
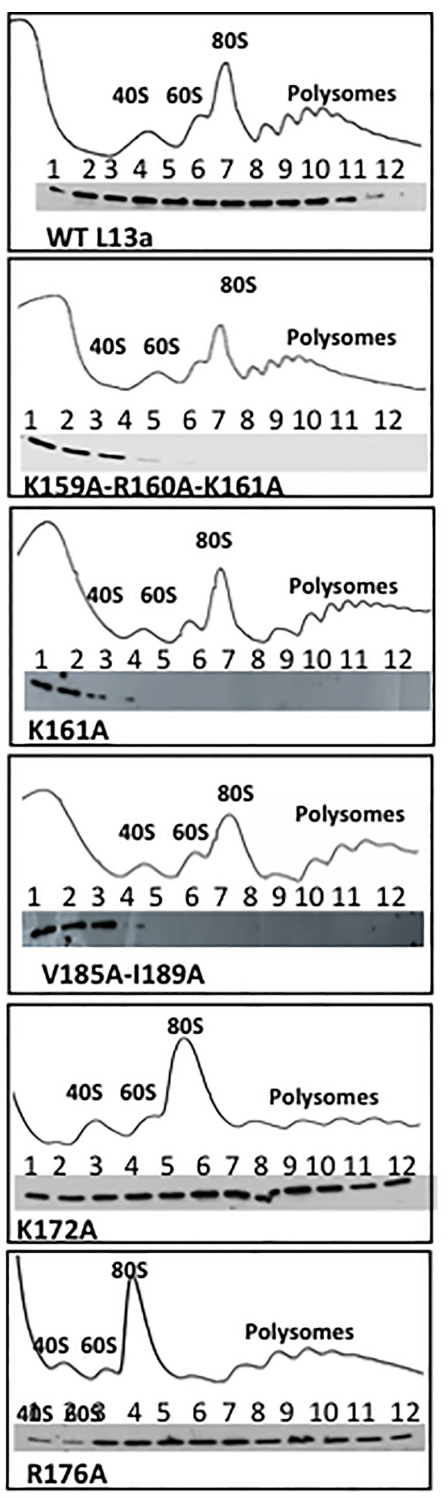

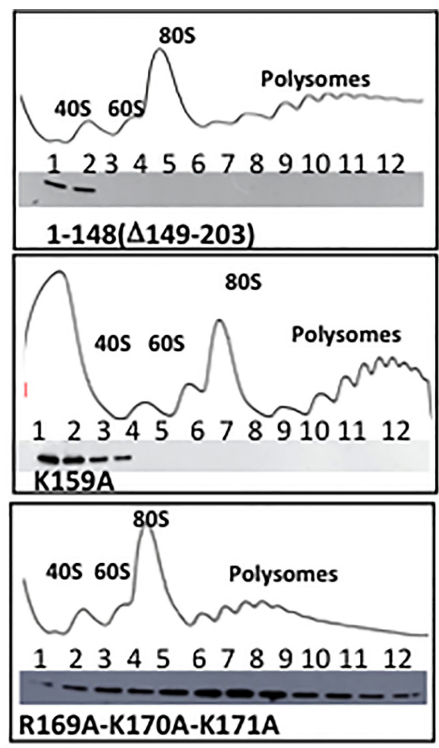

R169A-K170A-K171A
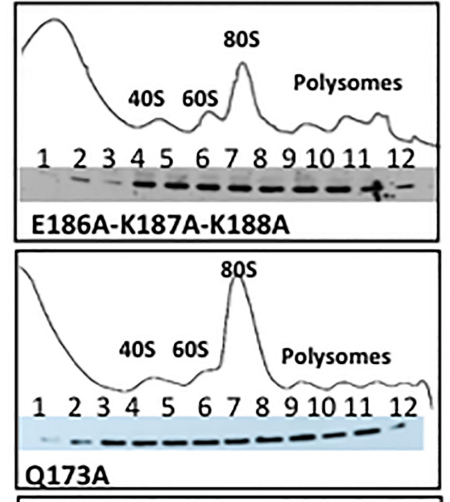

Q173A

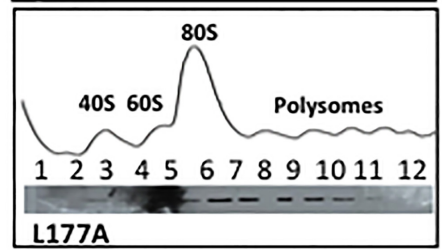

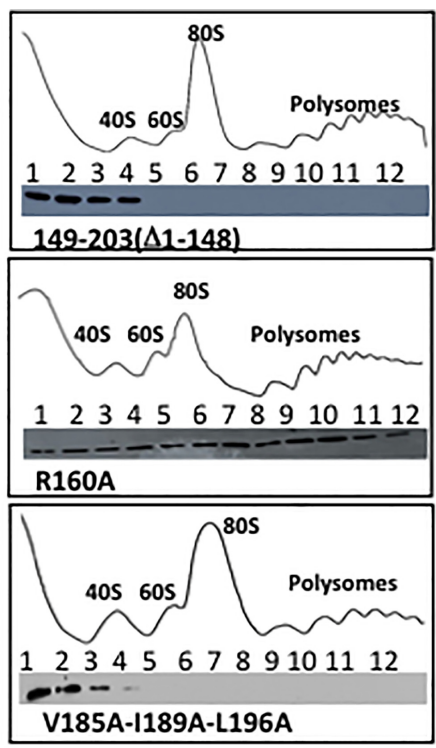
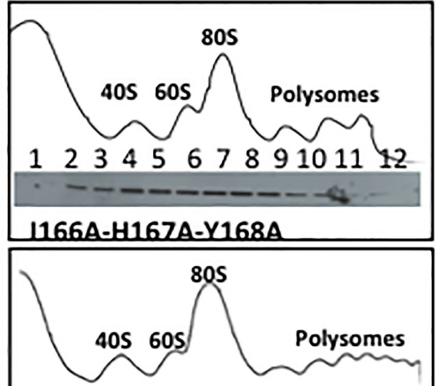

$\begin{array}{lllllllllll}1 & 2 & 3 & 4 & 5 & 6 & 7 & 8 & 9 & 1011 & 12\end{array}$

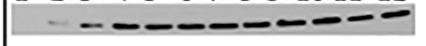

M175A

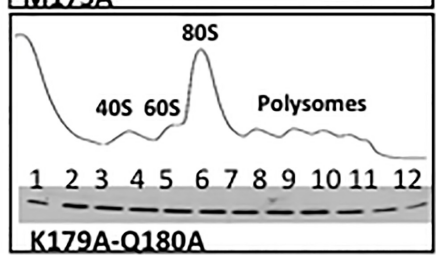

Individual absorption profiles showing ribosome fractionations for all L13a variants

B

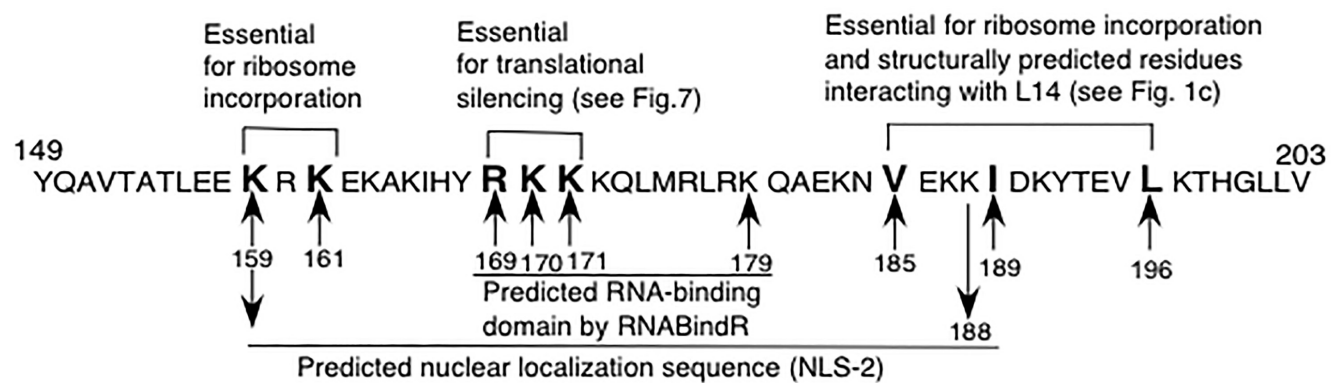

\section{Eukaryotic-specific C-terminal extension of L13a showing functional amino acid residues}

FIGURE 2. The eukaryotic-specific carboxy-terminal extension of L13a harbors amino acid residues required for its ribosomal incorporation. (A) Density gradient centrifugation cosedimentation analysis of $\mathrm{L} 13 \mathrm{a}$ variants. Absorption profiles during ribosome fractionations of individual L13a variants are shown. HA-tagged L13a (wild-type [WT] or mutant variants as indicated at the bottom of each profile) was expressed in HEK $293 T$ cells. Ribosomal fractions (\#1-12) were resolved by sucrose density gradient centrifugation (10\% to $50 \%$ ) and analyzed by immunoblotting with anti-HA antibody. The sedimentation of 40S, 60S, and $80 \mathrm{~S}$ ribosome subunits and polyribosomes is indicated at the top of the figure. (B) Schematic diagram of the human L13a amino acid sequence indicating residues shown to be critical for ribosomal incorporation based on the results shown in $A$ along with other predicted and experimentally determined features. 


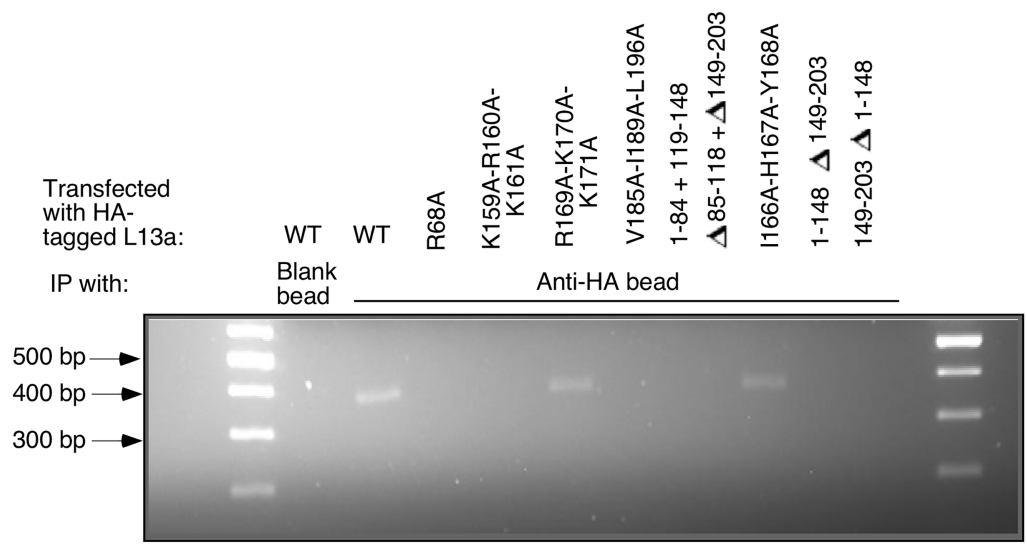

RT-PCR with $28 S$ rRNA-specific primers

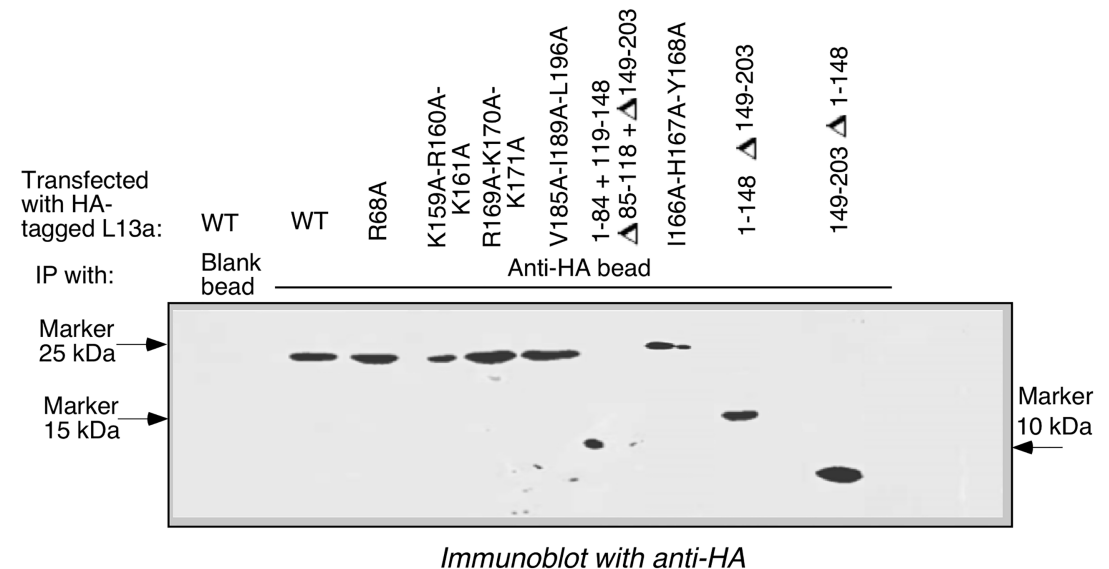

FIGURE 3. Association of $L 13 a$ and its mutant variants with $28 \mathrm{~S}$ rRNA. Lysates prepared from HEK 293T cells expressing HA-tagged L13a (WT or mutant as indicated above each lane) were used for immunoprecipitation with anti-HA-coated agarose beads (or nonantibody-coated blank beads as a control, first lane from left). (Top) Total RNA was extracted from the immunoprecipitates and analyzed by RT-PCR with primers specific for $28 \mathrm{~S}$ rRNA. RT-PCR products were visualized on an ethidium bromide-stained agarose gel. (Bottom) Equal volumes of the immunoprecipitate from each reaction were run on an SDS-PAGE gel and immunoblotted with anti-HA antibody to confirm the presence of equivalent amounts of HAtagged protein.

terminal extension deleted), 149-203 ( $\Delta 1-148$, with entire amino-terminal globular domain deleted), Lys159AlaArg160Ala-Lys161Ala, Val185Ala-lle189Ala-Leu196Ala, Arg59Ala-Lys60Ala-Lys61Ala (Fig. 4) and 1-84+119203 ( $\Delta 85-118$, internally deleted NLS-1), 1-84 + 119-148 ( $\Delta 85-118$ and $\Delta 149-203$ internally deleted NLS-1 and NLS-2) (Fig. 5) translocate into the nucleus. Surprisingly, all of the nuclear translocation-competent $\mathrm{L} 13 \mathrm{a}$ variants (except 149-203; $\Delta 1-148$, amino-terminal part deleted, Fig. 4) failed to translocate into the nucleolus. These variants were spread throughout the nucleus and failed to colocalize with nucleolin, thus showing their defects in nucleolar translocation (Figs. 4, 5). On the other hand, the ribosomal incorporation competent $\mathrm{L} 13 \mathrm{a}$ mutant Arg169Ala-Lys170Ala-Lys171Ala (just like the WT protein) was able to completely translocate into the nucleolus (Figs. $4,5)$. The overview of all the mutations and their consequences on ribosomal incorporation, nuclear, and nucleolar translocation have been summarized in Supplemental Table 1.

Nucleolin is a nucleolar protein, which is localized in the granular and dense fibrillar region of the nucleolus where ribosome assembly and rRNA processing takes place and plays a significant role in these processes, however nucleolin is not detectable in mature cytoplasmic ribosomes (Chen and Huang 2001; Boisvert et al. 2007; Pelletier et al. 2018). Nucleolin also interacts with a number of large and small subunit ribosomal proteins but the role of these interactions in the ribosomal incorporations of ribosomal proteins is far from clear (Bouvet et al. 1998). Our immunofluorescence data showed that most of the incorporation defective (except L13a 149-203 $\Delta 1-$ 148), but not the incorporation competent L13a mutants, failed to colocalize with nucleolin (Figs. 4, 5). Therefore, we have tested whether incorporation defective mutants failed to interact with nucleolin. As such, expressed HA-tagged L13a proteins were immunoprecipitated from HEK 293T cells using ant-HA antibody-conjugated beads and the L13a-bound coimmunoprecipitated nucleolin was detected by immunoblot using anti-nucleolin antibody. Previously identified ribosome incorporation defective, but nucleolin binding competent Arg68Ala mutant (Das et al. 2013), was used as a positive control alongside with a WT L13a. Wild-type protein, Arg68Ala L13a mutant and the incorporation competent mutants Arg169Ala-Lys170Ala-Lys171Ala, Ile166Ala-His167Ala-Tyr168A and Glu186A-Lys187AlaLys188Ala showed association with nucleolin (Fig. 6A). However, the incorporation defective mutants, Lys159Ala-Arg160Ala-Lys161Ala, Val185Ala-lle189AlaLeu196Ala and 1-84+119-148 ( $\Delta 85-118$ and $\Delta 149-203$ internally deleted NLS-1 and NLS-2) were not found to be associated with nucleolin (Fig. 6A). Surprisingly, we found that only the eukaryote-specific carboxy-terminal extension fragment 149-203 ( $\Delta 1-148)$ was sufficient to drive its nucleolar translocation (Fig. 4) and association with nucleolin (Fig. 6A). This fragment alone, however, failed to incorporate into the ribosome (Fig. 2). The inputs of nucleolin and the immunoprecipitated L13a variants as a control are presented in Figure 6A (lower panel) and Figure 6B, respectively. 
WT L13a

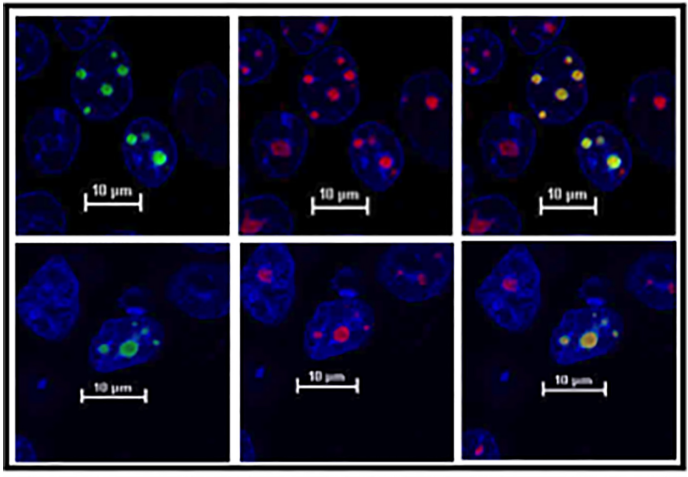

L13a (Green) Nucleolin (Red) Merge+ DAPI

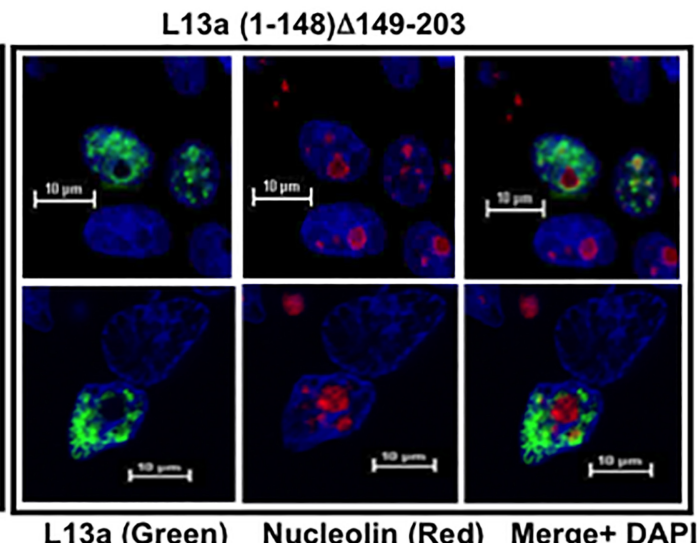

L13a (149-203) $\Delta 1-148$

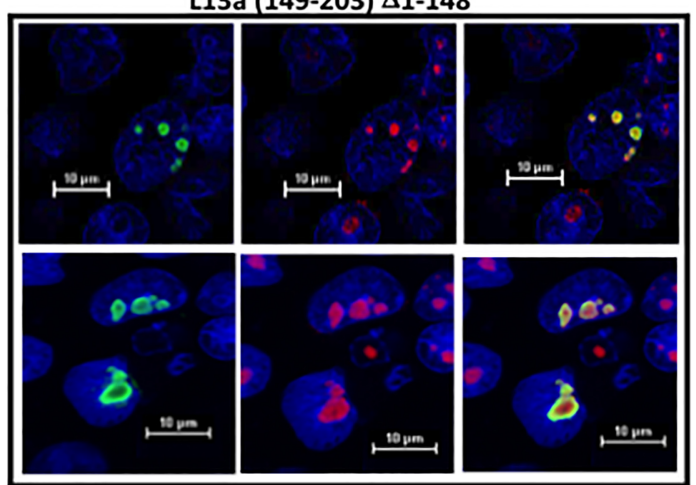

L13a (Green) Nucleolin (Red) Merge+ DAPI

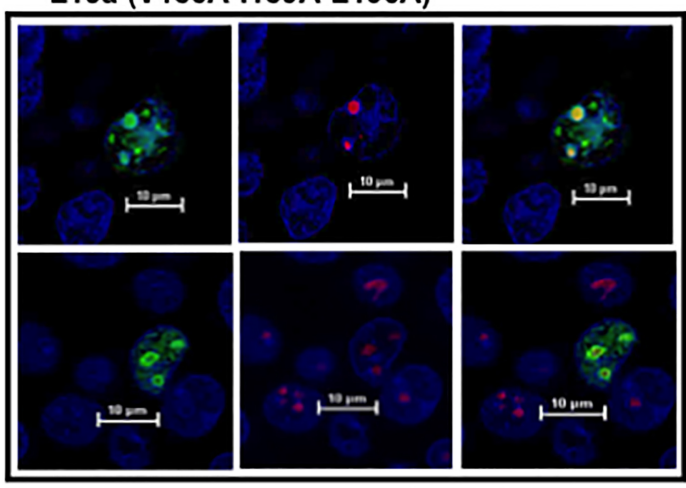

L13a (Green) Nucleolin (Red) Merge+ DAPI

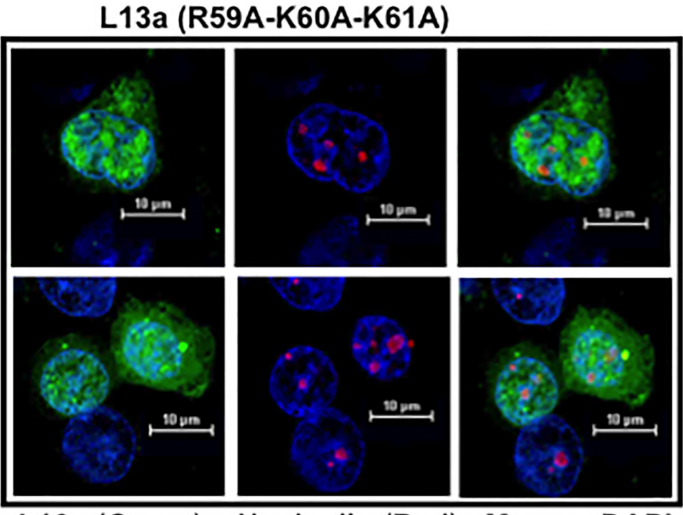

L13a (Green) Nucleolin (Red) Merge+ DAPI

FIGURE 4. Nuclear and nucleolar localization of L13a and its mutant variants. HEK 293T cells were transfected with constructs directing expression of HA-tagged L13a (WT or deletion mutants [1-148] and [149-203] or triple point mutants Lys159Ala-Arg160Ala-Lys161Ala, Val185AlaIle189Ala-Leu196Ala, and Arg59Ala-Lys60Ala-Lys61Ala as indicated above each set of six images). Cells were fixed $24 \mathrm{~h}$ post-transfection, stained with anti-HA (green), anti-nucleolin (red; a marker of the nucleolus), and DAPI (blue; to visualize nuclei), and viewed under a fluorescence microscope.

Together, these data suggest two crucial characteristics of $L 13$ a regarding its ribosomal incorporation, (i) association of L13a with nucleolin and nucleolar translocation is a necessary prerequisite, but not sufficient for its incorporation into the ribosome, and (ii) nuclear translocation of this protein is more refractory to the amino acid alterations compared to the nucleolar translocation.
L13a Arg169-Lys170-Lys171 residues of the eukaryote-specific carboxy-terminal extension are critically important for GAIT-mediated translational silencing

As described in the Introduction, mammalian L13a has an important physiological function outside of its role as a 
L13a (1-84 +119-203) 85-118 NLS1 deleted)

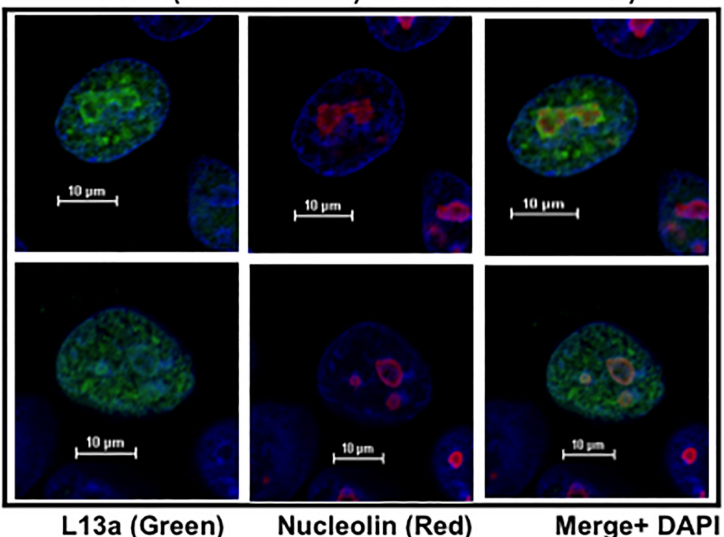

L13a (1-84 +119-148) 85-118 \& 149-203 NLS1 \& NLS2 deleted

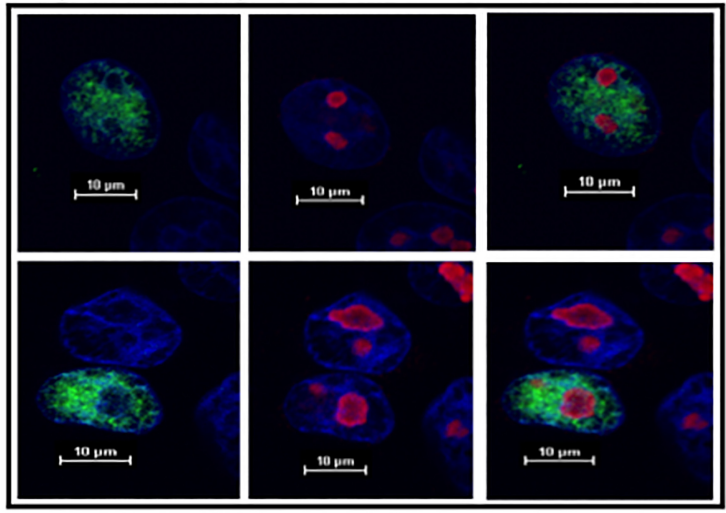

L13a (Green) Nucleolin (Red) Merge+ DAPI

L13a (R169A-K170A-K171A)

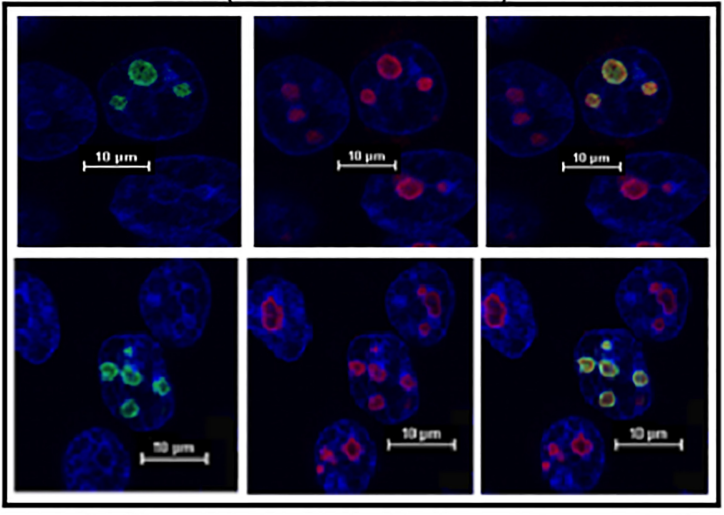

L13a (Green) Nucleolin (Red)

Merge+ DAPI

FIGURE 5. Nuclear and nucleolar localization of L13a variants lacking predicted NLSs or residues important for translational silencing. Nuclear and nucleolar localization of additional L13a variants (1-84 + 119-203; predicted NLS1 deleted), (1-84 + 119-148; predicted NLS1 and NLS2 deleted), and K169A-K170A-K171A (translational silencing incompetent, see Fig. 7) was analyzed by immunofluorescence as described in Figure 4.

ribosomal component. Under certain conditions, L13a becomes phosphorylated, released from the 605 ribosomal subunit, and incorporated into a complex of proteins (GAIT) that mediates translational silencing of a number of mRNAs encoding inflammatory proteins (Vyas et al. 2009). This has been validated as an important endogenous mechanism for controlling inflammation in vivo (Poddar et al. 2013, 2016; Basu et al. 2014). While L13a is an essential component of the GAIT complex (Sampath et al. 2004) many details of its role in this translational silencing mechanism are not fully understood. Therefore, in addition to examining the eukaryote-specific carboxyl terminus of L13a with respect to its contribution to ribosomal incorporation of the protein (see above), we also looked at the importance of this domain for GAIT-mediated translational silencing.

To do this, we used a previously established in vitro translation assay in which the addition of purified L13a protein results in reduced translation of a reporter RNA construct containing a GAIT sequence element. To generate the reporter construct, GAIT element from the human $\mathrm{Cp}$ mRNA (Sampath et al. 2003) were inserted into the 3'UTR of the luciferase mRNA. T7gene10 RNA (lacking a GAIT element) is included in each reaction as a control for translation efficiency and silencing specificity. In vitro translation of these constructs is performed using rabbit reticulocyte lysate (RRL) in the presence of ${ }^{35} \mathrm{~S}$-methionine so that translation products can be visualized by SDS-PAGE/ autoradiography. To test for GAIT-mediated translation silencing, recombinant $\mathrm{L} 13 \mathrm{a}$ (WT or variants engineered with mutations in the eukaryote-specific carboxyl terminus) was expressed in baculovirus-infected insect cells as previously described $(16,19,29)$ and added to the in vitro translation reactions.

As expected, the addition of WT L13a to this system inhibited translation of the GAIT element-containing luciferase RNA, but had no effect on the translation of the control T7gene10 RNA (without a GAIT element) present in the same reaction (Fig. 7A). No inhibition of translation was observed when luciferase RNA without GAIT element was used (data not shown). Next, we compared the activity of a number of different L13a deletion and point mutants to that of the WT protein in this in vitro translation system. As shown in Figure 7A and summarized in Figure 7B, L13a deletion mutants (1-195) $\Delta 196-203,(1-180) \Delta 181-$ 203 and (149-203) $\Delta 1-148$ retained activity in the GAITmediated translational silencing assay, while deletion mutants (1-162) $\Delta 163-203$ and (1-148) $\Delta 149-203$ did not. These results identified the region of the L13a eukaryote-specific carboxy-terminal extension from Tyr149 to Val203 (Fig. 7C) as essential for translational silencing.

In order to pinpoint the specific residues within this region that are critical for this silencing mechanism, we generated additional L13a variants containing single, double and triple point mutations (Fig 7C). When added to our in vitro translation system, L13a mutant variants Y149AQ150A， K159A-R160A-K161A， E162A-K163A， K172A， M175G, R176A, R178A, Q180A, K179A-Q180A, V185AI189A, and V185A-I189A-L196A were comparable to 
A

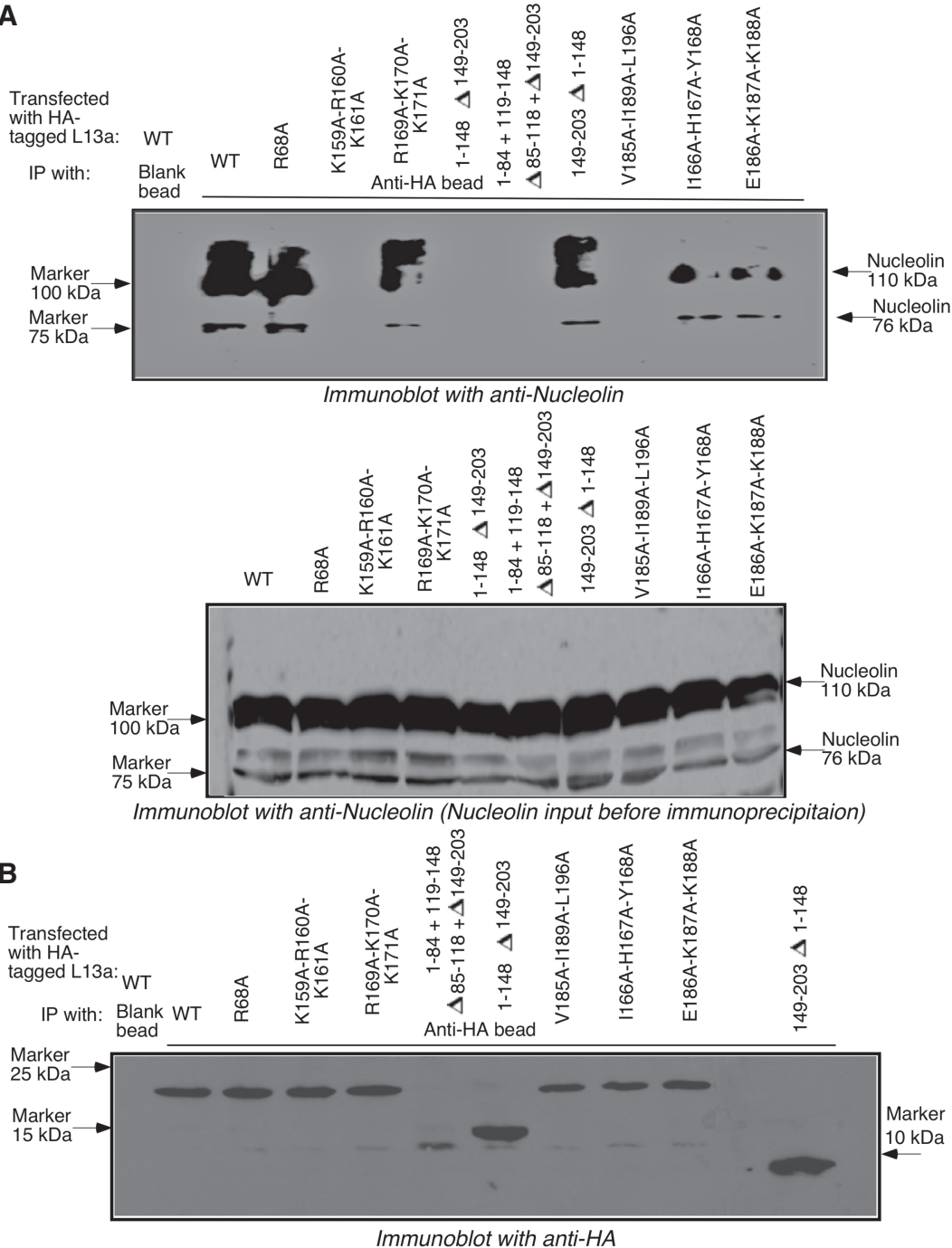

FIGURE 6. In vivo association of L13a and its mutant variants with nucleolin. (A) Lysates of HEK 293T cells expressing recombinant HA-tagged L13a (WT or mutant as indicated above each lane) were subjected to immunoprecipitation with anti-HA-coated beads (or nonantibodycoated blank beads as a control) as described for Figure 3. The immunoprecipitates were run on SDS-PAGE gels and immunoblotted with antinucleolin antibody (top panel), inputs of nucleolin before immunoprecipitation (bottom panel). (B) Immunoblot with anti-HA antibody.

WT L13a in their ability to induce translational silencing of the GAIT element-containing reporter luciferase mRNA (Fig. 7A). In contrast, the L13a triple mutant variant R169A-K170A-K171A failed to support GAIT elementdependent translational silencing (Fig. 7A). The quantitation of the result from the translational silencing experiment (with standard deviations) and all purified recombinant L13a variants used in this assay are shown in Supplemental Figure S3a,b, respectively. Together, these experiments reveal that a very short region of the L13a eukaryote-specific carboxy-terminal extension (Arg169Lys171) is essential for the extraribosomal function of L13a as a mediator of GAIT element-dependent transla- tional silencing. The precise mechanism by which this group of amino acids in L13a contributes to its translational silencing activity remains to be established. In addition, it remains possible that only one or two of the three identified amino acids is truly essential for silencing and/or that there are additional important amino acids within the Tyr149 to Val203 region of the protein. These questions can be resolved by testing additional L13a variants with targeted point mutations for GAIT element-dependent translational silencing.

\section{DISCUSSION}

Determination of the mechanisms that control the incorporation of L13a into ribosomes is important not only for improving our understanding of the complexities of ribosome biogenesis and function, but also for gaining insight into the unique extraribosomal function of L13a as a central molecule in a translational silencing pathway that is critical for the resolution of inflammation. Phosphorylation-induced release of $\mathrm{L} 13$ a from the $60 \mathrm{~S}$ ribosome is necessary for the protein to assemble into the complex that mediates GAIT element-dependent translational silencing (Mazumder et al. 2003). Thus, an understanding of how L13a becomes incorporated into ribosomes may also reveal aspects of the mechanisms that drive its ribosomal release for translational silencing. With this in mind, we aimed to map regions of L13a critical for its ribosomal incorporation and for GAIT elementmediated translational silencing. Our previous work identified Arg68 in the amino-terminal globular domain of human L13a as essential for its ribosomal incorporation (Das et al. 2013). Structural alignment of human L13a and E. coli L13 shows high conservation of the amino-terminal globular domains of these proteins (Supplemental Fig. $\mathrm{S} 1 \mathrm{~b})$. Considering the fundamental difference in the ribosome maturation and assembly process between prokaryotes and eukaryotes (Fromont-Racine et al. 2003; Henras et al. 2008), it is possible that human L13a may require additional amino acid residues for ribosomal incorporation. Therefore, in this study we focused our attention on its eukaryote-specific carboxy-terminal helical extension (Fig. 1; 
A

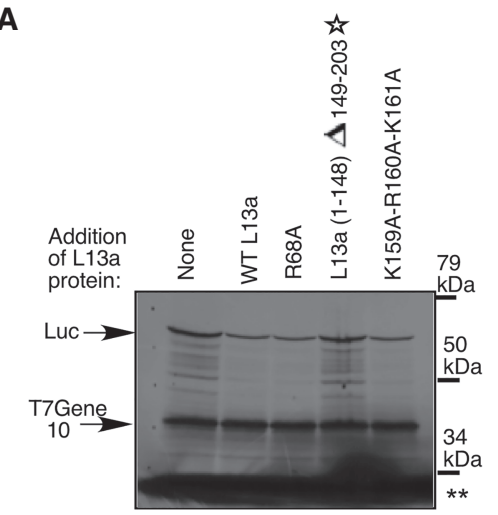

in vitro translation of $C R N A s$ in $R R L$

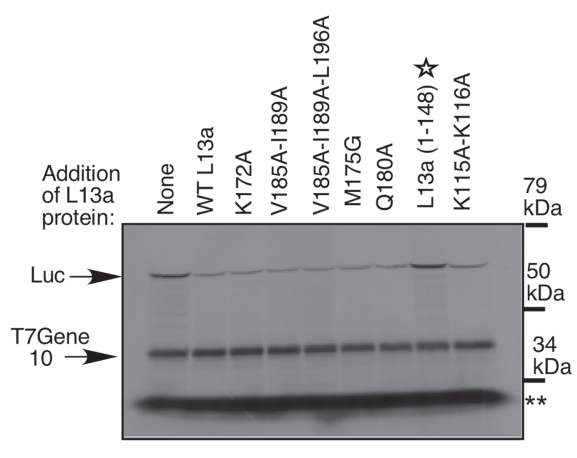

in vitro translation of $c R N A s$ in $R R L$

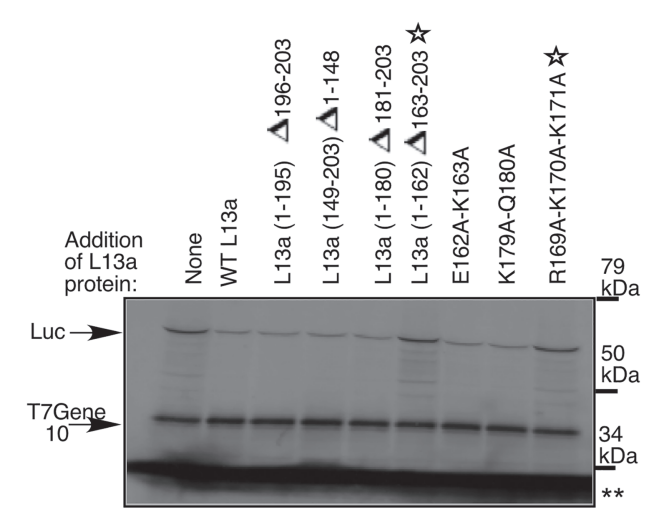

in vitro translation of $c R N A s$ in $R R L$

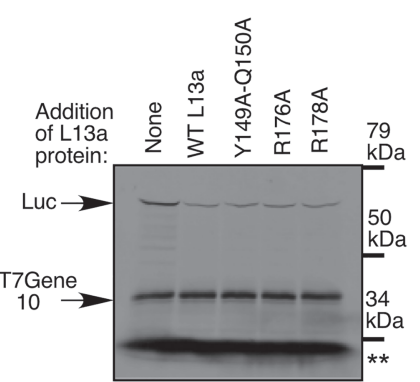

in vitro translation of $c R N A s$ in $R R L$

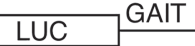

T7gene10

B

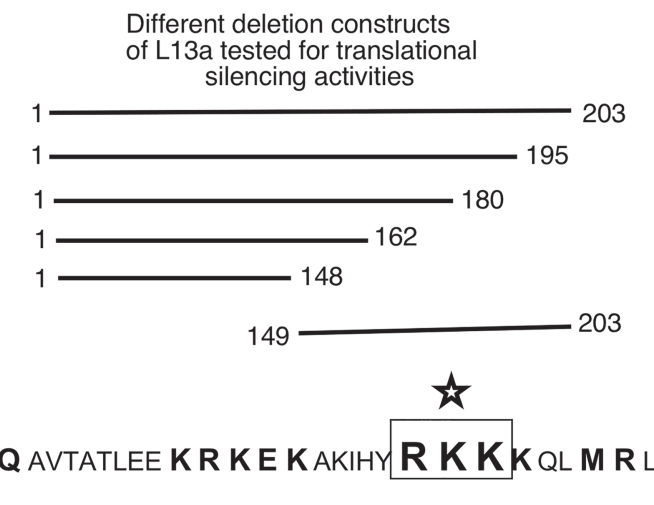

Abilities to retain translational silencing activities

YES

YES

YES

NO

NO

YES

\section{C 149}

FIGURE 7. The eukaryote-specific carboxy-terminal extension of L13a harbors amino acid residues required for GAIT element-mediated translational silencing. (A) Effect of L13a variants on in vitro translation of a GAIT element-containing luciferase reporter mRNA and a control mRNA lacking a GAIT element (T7 gene 10). In vitro translation assays were performed as described in Materials and Methods using RRL and purified Histagged L13a (WT or the indicated deletion or point mutants) produced in a baculovirus expression system. $\mathrm{S}^{35}$-radiolabeled proteins were visualized by $10 \%$ SDS-PAGE followed by autoradiography. L13a variants that did not induce GAIT element-dependent translational silencing are marked with a star. The double asterisk at the bottom of each gel shows the front of migration. (B) Schematic illustration of the L13a deletion constructs tested for translational silencing activity and a summary of the results. (C) Amino acid sequence of human L13a from Tyr149 to Val203 with residues tested by point mutations shown in bold font. The three amino acid region identified as essential for translational silencing is indicated by a box with a star.

Supplemental Fig. S1). This region harbors a predicted NLS (Lys159-Lys188; NLS-2) and a predicted RNA-binding domain (Arg169-Lys179) lying within the NLS (Fig. 1A). It also contains a region structurally predicted to be in interaction (via Val185, lle189, and Leu196) with ribosomal protein L14 (Fig. 1C).
As discussed further below, our study does not provide any evidence that the predicted NLS in the eukaryotespecific carboxy-terminal extension of L13a plays a role in L13a translocation into the nucleus. However, several residues within the same region were shown to be essential for incorporation of $\mathrm{L} 13 \mathrm{a}$ protein into mature 
ribosomes. These residues include Lys159, Lys161, Val185, and Ile189 (Fig. 2), with the latter two being predicted to be involved in the interaction between the carboxy-terminal helices of $\mathrm{L} 13 \mathrm{a}$ and another ribosomal protein, L14. We propose that these additional amino acids within the eukaryote-specific carboxy-terminal extension of L13a may be needed for L13a ribosomal incorporation due to its larger size compare to its prokaryotic counterpart. Additional contact points (such as with L14 or rRNA) could facilitate recruitment and incorporation of the longer protein. While it has been speculated that productive UL13-L14 interaction might be important for uL13 incorporation into ribosomes (Ben-Shem et al. 2011; Klinge et al. 2011) other studies in yeast did not support this notion (Espinar-Marchena et al. 2016). Deletion of the last 18 amino acids of yeast L16B had no effect on 605 ribosomal subunit production (Espinar-Marchena et al. 2016), while longer truncations impaired ribosomal biogenesis (Ohmayer et al. 2015). We note however that while mammalian $\mathrm{L} 13 \mathrm{a}$ and yeast L16 share almost identical structures (Supplemental Fig. S4), the amino acid composition of the eukaryote-specific carboxy-terminal extension is not identical and differs between the two (Supplemental Fig. S5). The molecular environment (i.e., set of contacts those proteins, and, especially, their eukaryote-specific carboxy-terminal extensions form with rRNA) also differs (Supplemental Fig. S6). These features may in part explain the observed differences between the yeast and mammalian systems; however, more in-depth studies of L13a-L14 interaction may provide additional insight into the mechanism of L13a ribosomal incorporation and reveal the exact origin of the differences observed between mammalian and yeast systems.

In eukaryotes, ribosomal proteins are synthesized in the cytoplasm and then translocate into the nucleus and eventually into the nucleolus where they are incorporated into nascent ribosomes. Therefore, it is generally assumed that all ribosomal proteins must harbor NLSs. Structurebased studies of the universally conserved ribosomal protein S12 from eukaryotes and prokaryotes, combined with cellular immunofluorescence experiments, revealed several functional NLSs located in the nonglobular extension of the rRNA-binding domain, spanning conserved as well as nonconserved eukaryote-specific regions (Melnikov et al. 2015). A similar situation appeared to exist for human ribosomal protein L13a given the in silico predictions made by NLS mapper and NLStradamus (Fig. 1) indicating one NLS spanning Arg84 to Val118 within the structurally conserved amino-terminal globular domain of the protein and another spanning Lys159 to Lys188 within the eukaryote-specific carboxy-terminal helical extension (Fig. 1; Supplemental Fig. S1). However, our immunofluorescence-based analysis of the in vivo subcellular localization of a relatively large set of $\mathrm{L} 13$ a mutant variants failed to identify a functional NLS in the protein (Figs. 4, 5). Even complete deletion of both NLS-1 and NLS-2 did not abrogate L13a translocation into the nucleus (Fig. 5). This suggests that L13a may utilize a nonconventional mechanism/ signal sequence (not predictable by current bioinformatics platforms) to enter the nucleus. Work by others on L12, L5, $L 11$, and L23a showed that translocation of ribosomal proteins into the nucleus relies on association of these proteins with nuclear import receptors of the importin $\beta$ / karyopherin family of proteins and that different ribosomal proteins use different importins that recognize diverse NLS sequences (Rout et al. 1997; Plafker and Macara 2002; Kressler et al. 2012). Future studies on the specific nuclear import receptors used by L13a may uncover the mechanism and signal sequence(s) responsible for its nuclear import.

Some previous studies have suggested that NLSs of ribosomal proteins may not only promote nuclear translocation of these proteins, but may also directly participate in establishing contacts with rRNA promoting ribosomal incorporation of the protein (Melnikov et al. 2015). The presence of a predicted RNA binding site within NLS-2 in the eukaryote-specific carboxy-terminal extension of $\mathrm{L} 13 \mathrm{a}$ (Fig. 1) is consistent with this being the case for L13a. Surprisingly, however, none of the ribosome incorporation-defective $\mathrm{L} 13 \mathrm{a}$ variants tested in our study showed any defect in nuclear translocation (Figs. 4, 5). This suggests that distinct sets of residues in L13a guide its nuclear translocation and rRNA-binding/ribosomal incorporation.

Notably, while all of the ribosome incorporationdefective mutants retained the ability to translocate into the nucleus, all except one (149-203 [ $\Delta 1-148]$, consisting of the carboxy-terminal extension alone) failed to move from the nucleus to the nucleolus. This finding strongly implies that shared or overlapping residues of the protein control its nuclear to nucleolar translocation and ribosomal incorporation. A clear conclusion of this study is that while nuclear translocation of a ribosomal protein may be a prerequisite for its incorporation into ribosomes, it is not sufficient on its own to result in nucleolar translocation or ribosomal incorporation. Why nuclear translocation of $\mathrm{L} 13 \mathrm{a}$ is relatively unaffected by widespread amino acid changes compared to nucleolar translocation remains unclear. However, one possibility is that L13a may have a currently unknown function in the nucleus that is more important than its role in ribosome biogenesis in the nucleolus. This could be consistent with our earlier studies in human cells showing that the protein synthesis function of ribosomes does not require L13a (Chaudhuri et al. 2007). This observation is in contrast to the yeast system, where it has been shown that L16 is indispensable for cellular growth and ribosome function (Ohmayer et al. 2015; Espinar-Marchena et al. 2016).

Eukaryote-specific extensions of eukaryotic ribosomal proteins are currently the subject of intensive investigation. They are pivotal in ribosome biogenesis through their 
involvement in specific pre-rRNA processing reactions, formation of binding sites for ribosome assembly factors, and promotion of nuclear localization of ribosomal proteins (due to presence of NLSs) and also to contribute to specific functions of mature ribosomes (i.e., specific steps in protein synthesis) (Fernández-Pevida et al. 2016). L13a, apart from being a ribosomal protein, also has an extraribosomal function as an essential component of the GAIT protein complex (Mazumder et al. 2003; Sampath et al. 2004). The results of this study show that the L13a eukaryote-specific carboxy-terminal extension (Tyr 149 to Val 203) alone can support GAIT element-mediated translational silencing and that mutation of three positively charged amino acids in this region (Arg169-Lys170-Lys171) to hydrophobic alanines completely abrogates silencing (Fig. 7). Thus, our study revealed the essential role of the eukaryote-specific carboxy-terminal extension of the mammalian L13a in GAIT-mediated translational silencing process not noted before. However, this type of translational control has not been reported in yeast. Given our earlier work demonstrating the physiological significance of GAIT-mediated translational silencing for resolution of inflammation (Poddar et al. 2013, 2016; Basu et al. 2014), it is possible that this eukaryote-specific carboxy-terminal extension further evolved in multicellular higher eukaryotes (mammals) to acquire additional role in the inflammation process caused by immune cells. The conservation of an Arg169-Lys170Lys171 triad among mouse, human, and bovine species but not among the lower eukaryotes (Supplemental Fig. S5) is consistent with this hypothesis. The alignment of uL13s across seven eukaryotic species, namely, C. elegans, S. cerevisiae, P. mariana, D. melanogaster, M. musculus, $H$. sapiens, and B. taurus are shown (Supplemental Fig. S5). This comparison shows that the amino-terminal (globular) part of the protein is more conserved than the eukaryotespecific carboxy-terminal extension fragment comprising last $\sim 55$ amino acid residues. However, we also note that the conservation of the eukaryote-specific extension within the higher eukaryotes (Human, Mouse, Bovine, Drosophila) is much higher than in lower eukaryotes (e.g., Yeast and Worms). This alignment also shows that Arg68 (Das et al. 2013) and Lys159, Arg160, Lys161 (this manuscript) residues important for ribosome incorporation are highly conserved among all of these seven species from Yeast to Human, but the Arg169-Lys170-Lys171 triad responsible for translational silencing is only conserved among higher eukaryotes (mammals), for example, human, mouse, bovine (Supplemental Fig. S5). Obviously, the eukaryotespecific carboxy-terminal extension fragment has further evolved from yeast to mammals.

Our ability to reconstitute GAIT-mediated translational silencing using only the eukaryote-specific carboxy-terminal extension of L13a raises the questions of how exactly the carboxy-terminal extension contributes to GAIT complex assembly and what is the precise role of DAP
Kinase-ZIP Kinase axis-mediated Ser77 phosphorylation (Mukhopadhyay et al. 2008) in the process of L13a release from the ribosome and assembly into the GAIT complex. Since unphosphorylated full-length human L13a expressed in bacterial cells cannot support GAIT-mediated translational silencing (Mazumder et al. 2003), but the carboxy-terminal extension alone (which does not contain Ser77) can, we hypothesize that Ser77 phosphorylation may have a dual function. Phosphorylation at Ser77 may cause conformational change in the protein and as a consequence the amino acid residue(s) providing the contacts with the rRNA, for example, Arg68 (Das et al. 2013) and Lys159-Arg160-Lys161 (Fig. 2; Supplemental Fig. S6) could fail to anchor with the rRNA thus causing the release. In addition to driving the release of L13a from the ribosome, the phosphorylation-induced conformational change in the protein could allow the carboxy-terminal helical extension to engage in GAIT complex assembly (see proposed model in Supplemental Fig. S7). This possibility is supported by the spatial proximity of a number of negatively charged amino acids to Ser77 in the folded L13a protein (Supplemental Fig. S7). As such, it is reasonable to propose that addition of a negatively charged phosphate group at Ser77 could cause electrostatic repulsion leading to movement of the carboxy-terminal helix away from the amino-terminal globular domain and that this "opening up" of the protein could expose residues critical for L13a assembly into the GAIT complex. While this hypothesis provides an explanation for the difference in silencing activity of bacterially expressed unphosphorylated fulllength human L13a versus the carboxy-terminal extension alone (see above), its verification will require additional comparative experimental structural analysis of phosphorylated and unphosphorylated L13a.

Of practical importance, our finding that mutation of Arg169-Lys170-Lys171 eliminates the GAIT elementmediated translational silencing activity of L13a without affecting its incorporation into ribosomes presents the possibility of generating a whole-body knock-in mouse model to further examine the physiological function of L13a-mediated translational silencing on a systemic level. While a macrophage-specific L13a knockout mouse model was successfully generated and used to demonstrate the physiological role of L13a-mediated translational silencing in resolution of inflammation (Poddar et al. 2013, 2016; Basu et al. 2014), it has not been possible to generate systemic/total body L13a knockout mice due to early embryonic lethality (data not shown). This lethal phenotype clearly indicates that L13a has important physiological functions that have yet to be uncovered, so having the means to address its translational silencing activity on a systemic level would be a valuable step forward.

Overall, the results of the current study suggest that the eukaryote-specific carboxy-terminal extension of mammalian L13a might have evolved to enhance its ribosomal 
incorporation and allow its function in GAIT-mediated translational silencing. These findings add to our understanding of the complex processes of ribosome biogenesis and function, as well as the unique extraribosomal function of L13a. This study also shows that evolutionary conserved proteins and their particular fragments could adopt different functions depending on the environment and the host organism.

\section{MATERIALS AND METHODS}

\section{Structural and sequence analysis of human and yeast L13a (L16A) and the molecular environment of the proteins on the ribosome surface}

Visualization and analysis of L13a and L14 structures were performed using CryoEM data of the human ribosome at $3.6 \AA$ resolution (Protein Data Bank code 5T2C) and X-Ray data of the yeast ribosome at $3.0 \AA$ \& resolution (Protein Data Bank code 4V88). Ribosome and protein structures were visualized and analyzed using Swiss-Pdb Viewer V4.1.0 and/or PyMOL v1.5.0.5. The PyMOL interface residues script was used to select interface residues involved in the interaction between L13a and L14. Structural alignment of the human L13a and a yeast homolog (L16A) was done using the interactive fit (all atom) alignment option of the SwissPdb Viewer. Alignments of amino acid sequences of ribosomal proteins were done using Clustal Omega (1.2.4) (https://www .ebi.ac.uk/Tools/msa/clustalo/) that uses seeded guide trees and hidden Markov model (HMM) profile-profile techniques to generate alignments between three or more sequences.

\section{Cell culture}

HEK 293T cells were maintained in DMEM medium supplemented with $10 \%$ fetal bovine serum (FBS), $2 \mathrm{mM}$ glutamine, $1 \%$ penicillin and streptomycin at $37^{\circ} \mathrm{C}$ and $5 \% \mathrm{CO}_{2}$. Sf9 cells were maintained in Sf-900 II SFM (1 $\times$ ) serum-free media in a nonhumidified incubator at $27^{\circ} \mathrm{C}$.

\section{Generation and expression of L13a cDNA constructs}

Hemagglutinin (HA)-tagged L13a cDNAs were generated by cloning the human WT L13a cDNA or its different mutant variants in-frame with the HA tag in the pcDNA3.1(+) mammalian expression vector (Invitrogen) using flanking BamHI and Notl restriction sites. The resulting constructs were transfected into HEK 293T cells using Lipofectamine 2000 according to the manufacturer's protocol (Thermo Fisher Scientific). His-tagged recombinant L13a bacmid DNAs were generated by cloning human L13a cDNAs (WT or different mutant variants) into pFastBac HT A vector using EcoRI and Sall restriction sites. The resulting constructs were introduced into Max efficiency DH10bac competent cells using the Bac-to-Bac Baculoviral Expression System kit according to the manufacturer's protocol (Invitrogen). Mutations in the cloned cDNAs were generated by site-directed mutagenesis using the Quick Change II Site-Directed Mutagenesis Kit (Agilent Technologies). L13a deletion mutants were generated by PCR of different length L13a fragments followed by cloning into pcDNA3.1(+) and pFastBac HT A. HA and His tags were placed at the amino terminus of the $\mathrm{L} 13 \mathrm{a}$ open reading frame.

\section{Ribosomal incorporation assay for L13a}

Wild-type and different mutant variants of HA-tagged L13a proteins were tested for their ability to cosediment with polyribosomes. HEK293T cells were transfected with either WT or mutant human L13a cDNAs cloned in pcDNA3.1(+). Eighteen hours post-transfection, polyribosomes were harvested from the transfected cells by our previously published method (Das et al. 2013). Briefly, transfected cells were treated with cycloheximide $(\mathrm{CHX})(100 \mu \mathrm{g} / \mathrm{mL})$ for $15 \mathrm{~min}$ at $37^{\circ} \mathrm{C}$, washed once and harvested in PBS containing $\mathrm{CHX}$ at $100 \mu \mathrm{g} / \mathrm{mL}$ concentration. Cells were lysed in polyribosome buffer $(100 \mathrm{mM} \mathrm{KCl}, 2.5 \mathrm{mM} \mathrm{MgCl}, 1$ $\mathrm{mM}$ DTT, $10 \mathrm{mM}$ HEPES $\mathrm{pH}$ 7.5, $100 \mu \mathrm{g} / \mathrm{mL}$ CHX) containing $0.1 \%$ Igepal-CA630 (NP-40), $50 \mathrm{U}$ of recombinant RNasin (Promega) and protease inhibitor cocktail (Roche). Cytoplasmic lysates (20 Optical density units) were layered over a $10 \%-50 \%$ linear sucrose gradient in polyribosome buffer and then centrifuged at 17,000 RPM in a Beckman SW32.1 Ti rotor for $18 \mathrm{~h}$ at $4^{\circ} \mathrm{C}$. Gradients were fractionated to get lighter RNP fractions $(40,60$, and $80 \mathrm{sec}$ ) and heavier polyribosomal fractions using an ISCO Gradient Fractionation System by monitoring the continuous UV absorption profile at $A_{254}$. The total protein in each fraction was recovered by TCA precipitation and analyzed by SDS-PAGE followed by immunoblotting with a rabbit polyclonal anti-HA antibody (Abcam).

\section{Analysis of nuclear and nucleolar localization of L13a by immunofluorescence}

HEK 293T cells were transfected with pcDNA3.1(+)-based constructs directing expression of HA-tagged WT or mutant human L13a. Twenty-four hours post-transfection, cells were fixed with methanol, permeabilized with $0.1 \%$ Triton X-100, and blocked in $1 \%$ BSA. Cells were then incubated with mouse monoclonal antibody against the HA tag (Abcam) or rabbit polyclonal antibody against human nucleolin (as an endogenous marker of nucleoli; Abcam) for $3 \mathrm{~h}$. After incubation with the primary antibodies, cells were washed with PBS and incubated with Alexa Fluor 488 (green) conjugated donkey anti-mouse antibody (Invitrogen; for anti-HA stained cells) or Alexa Fluor 594 (red) conjugated donkey antirabbit antibody (Invitrogen; for anti-nucleolin stained cells). All cells were costained with 4',6-diamidino-2-phenylindole (DAPI, blue) to visualize nuclei. After washing, the antibody-stained cells were subjected to confocal microscopy using a Nikon TE inverted fluorescent microscope.

\section{Determination of the GAIT element-dependent translational silencing activity of L13a using a cell- free in vitro translation system}

His-tagged recombinant WT and different mutant variants of $L 13 a$ proteins were expressed in Sf9 cells using the Bac-to-Bac baculoviral expression system following the manufacturer's protocol (Invitrogen). Briefly, Sf9 insect cells were transfected with WT or 
mutant L13a bacmids to generate baculoviral stocks. Sf9 cells were further infected with the baculoviral particles, lysed and His-tagged L13a proteins were purified using HisPur Ni-NTA purification Kit (Thermo Fisher Scientific). Purified proteins were tested using an in vitro translational silencing assay. Two hundred nanograms GAIT element bearing chimeric RNA transcript containing a GAIT element (Cap-Luc-GAIT-PolyA) and a cRNA transcript encoding T7 gene 10 (used as a loading and specificity control) were translated in rabbit reticulocyte lysate (RRL) in the presence of purified WT or mutant L13a proteins, $20 \mu \mathrm{M}$ methionine-free amino acid mixture, $20 \mu \mathrm{Ci}$ translation grade $\mathrm{S}^{35}$ methionine, and $40 \mathrm{U}$ of RNasin in a total volume of $50 \mu \mathrm{L}$ reaction at $30^{\circ} \mathrm{C}$ for $1 \mathrm{~h}$. An aliquot was resolved by 10\% SDS-PAGE. The gel was fixed, dried and radiolabeled bands were detected by autoradiography.

\section{In vivo association of L13a with $28 \mathrm{~S}$ rRNA}

HEK 293T cells were transfected with plasmids directing the expression of HA-tagged WT or mutant L13a proteins. Twentyfour hours post-transfection, cell lysates were prepared and subjected to immunoprecipitation using mouse anti-HA antibody coupled agarose beads (Sigma) or nonantibody-coupled beads as a control in the buffer $150 \mathrm{mM} \mathrm{NaCl}, 1 \mathrm{mM}$ EDTA, $1.5 \mathrm{mM}$ $\mathrm{MgCl}_{2}, 0.05 \%$ Triton X-100, 50 mM HEPES (pH 7.5) (Mazumder et al. 2003). Total L13a-bound RNA was extracted from the immunoprecipitates using TRIzol. The extracted RNA was reversetranscribed using random hexamers and reverse transcriptase (superscript) following the manufacturer's protocol (Invitrogen). The synthesized first-strand cDNA was subjected to reverse transcription-PCR (RT-PCR) using human 28S rRNA specific primer pairs. Forward primer sequence 5'-GAAGTTTCCCTCAGGATAGCT-3' and reverse primer sequence 5'-GCAGGTGAGTTGTTACAC ACT-3' were used. The PCR product of 355 base pairs was analyzed by agarose gel electrophoresis. Immunoblotting with anti$\mathrm{HA}$ antibody was used to confirm the presence of L13a in the immunoprecipitate.

\section{In vivo association of L13a with nucleolin}

HEK 293T cells were transfected with plasmids directing the expression of HA-tagged WT or mutant L13a proteins. Twentyfour hours post-transfection, cells were lysed and $100 \mu \mathrm{g}$ of lysates were immunoprecipitated with mouse anti-HA antibody coupled agarose beads (Sigma) or nonantibody-couple beads as a control in the buffer $150 \mathrm{mM} \mathrm{NaCl}, 1 \mathrm{mM}$ EDTA, $1.5 \mathrm{mM} \mathrm{MgCl}_{2}, 0.05 \%$ Triton X-100, 50 mM HEPES (pH 7.5). Ten micrograms of lysates before immunoprecipitation were used as an input for nucleolin. Total protein from the immunoprecipitates was subjected to SDSPAGE, followed by immunoblot analysis with mouse monoclonal anti-nucleolin antibody (Millipore, Catalog\# MABC587). To confirm the equal efficiency of immunoprecipitation in all of the samples immunoblot was also performed with rabbit anti-HA antibody (Abcam).

\section{SUPPLEMENTAL MATERIAL}

Supplemental material is available for this article.

\section{DATA DEPOSITION}

"Foldalign" (http://foldalign.ku.dk), "Swiss-Pdb viewer" (https:// spdbv.vital-it.ch), "PyMOL" (https://pymol.org/2/), "NLS mapper" (http://nls-mapper.iab.keio.ac.jp/cgi-bin/NLS_Mapper_form .cgi), and "NLStradamus" (http://www.moseslab.csb.utoronto .ca/NLStradamus/) are open source platforms.

\section{ACKNOWLEDGMENTS}

This work was supported by National Institutes of Health (NIH) grants HL79164 (to B.M.) and HL121779 (to A.A.K.), Shared instrumentation grant 1S10OD025252-01, and by the Cleveland State University Office of Research and the Center for Gene Regulation in Health and Disease (GRHD).

Author contributions: B.M. and R.K. conceived and designed the study. R.K. performed most of the experiments. A.A.K. performed the modeling of L13a, and B.M. and A.A.K. conducted analyses, interpreted results, and wrote the manuscript. All authors contributed to the final version of the manuscript.

Received March 11, 2019; accepted July 11, 2019.

\section{REFERENCES}

Basu A, Poddar D, Robinet P, Smith JD, Febbraio M, Baldwin WM III, Mazumder B. 2014. Ribosomal protein L13a deficiency in macrophages promotes atherosclerosis by limiting translation controldependent retardation of inflammation. Arterioscler Thromb Vasc Biol 34: 533-542. doi:10.1161/ATVBAHA.113.302573

Basu A, Jain N, Tolbert BS, Komar AA, Mazumder B. 2017. Conserved structures formed by heterogeneous RNA sequences drive silencing of an inflammation responsive post-transcriptional operon. Nucleic Acids Res 45: 12987-13003. doi:10.1093/nar/gkx979

Behrmann E, Loerke J, Budkevich TV, Yamamoto K, Schmidt A, Penczek PA, Vos MR, Bürger J, Mielke T, Scheerer P, et al. 2015. Structural snapshots of actively translating human ribosomes. Cell 161: 845-857. doi:10.1016/j.cell.2015.03.052

Ben-Shem A, Garreau de Loubresse N, Melnikov S, Jenner L, Yusupova G, Yusupov M. 2011. The structure of the eukaryotic ribosome at $3.0 \AA$ resolution. Science 334: 1524-1529. doi:10 $.1126 /$ science. 1212642

Boisvert FM, van Koningsbruggen S, Navascués J, Lamond Al. 2007. The multifunctional nucleolus. Nat Rev Mol Cell Biol 8: 574-585. doi:10.1038/nrm2184

Bouvet P, Diaz JJ, Kindbeiter K, Madjar JJ, Amalric F. 1998. Nucleolin interacts with several ribosomal proteins through its RGG domain. J Biol Chem 273: 19025-19029. doi:10.1074/jbc.273.30.19025

Chaudhuri S, Vyas K, Kapasi P, Komar AA, Dinman JD, Barik S, Mazumder B. 2007. Human ribosomal protein L13a is dispensable for canonical ribosome function but indispensable for efficient rRNA methylation. RNA 13: 2224-2237. doi:10.1261/rna.694007

Chen D, Huang S. 2001. Nucleolar components involved in ribosome biogenesis cycle between the nucleolus and nucleoplasm in interphase cells. J Cell Biol 153: 169-176. doi:10.1083/jcb.153.1.169

Das P, Basu A, Biswas A, Poddar D, Andrews J, Barik S, Komar AA, Mazumder B. 2013. Insights into the mechanism of ribosomal incorporation of mammalian L13a protein during ribosome biogenesis. Mol Cell Biol 33: 2829-2842. doi:10.1128/MCB.00250-13

Espinar-Marchena FJ, Fernández-Fernández J, Rodríguez-Galán O, Fernandez-Pevida A, Babiano R, de la Cruz J. 2016. Role of the yeast ribosomal protein L16 in ribosome biogenesis. FEBS $J$ 283: 2968-2985. doi:10.1111/febs. 13797 
Fernández-Pevida A, Martín-Villanueva S, Murat G, Lacombe T, Kressler D, de la Cruz J. 2016. The eukaryote-specific N-terminal extension of ribosomal protein S31 contributes to the assembly and function of $40 \mathrm{~S}$ ribosomal subunits. Nucleic Acids Res 44: 7777-7791. doi:10.1093/nar/gkw641

Fromont-Racine M, Senger B, Saveanu C, Fasiolo F. 2003. Ribosome assembly in eukaryotes. Gene 313: 17-42. doi:10.1016/S03781119(03)00629-2

Henras AK, Soudet J, Gérus M, Lebaron S, Caizergues-Ferrer M, Mougin A, Henry Y. 2008. The post-transcriptional steps of eukaryotic ribosome biogenesis. Cell Mol Life Sci 65: 2334-2359. doi:10 .1007/s00018-008-8027-0

Herold M, Nierhaus KH. 1987. Incorporation of six additional proteins to complete the assembly map of the $50 \mathrm{~S}$ subunit from Escherichia coli ribosomes. J Biol Chem 262: 8826-8833.

Kapasi P, Chaudhuri S, Vyas K, Baus D, Komar AA, Fox PL, Merrick WC, Mazumder B. 2007. L13a blocks 48S assembly: role of a general initiation factor in mRNA-specific translational control. Mol Cell 25: 113-126. doi:10.1016/j.molcel.2006.11.028

Klinge S, Voigts-Hoffmann F, Leibundgut M, Arpagaus S, Ban N. 2011. Crystal structure of the eukaryotic 605 ribosomal subunit in complex with initiation factor. Science 334: 941-948. doi:10 $.1126 /$ science. 1211204

Kressler D, Bange G, Ogawa Y, Stjepanovic G, Bradatsch B, Pratte D, Amlacher S, Strauss D, Yoneda Y, Katahira J, et al. 2012. Synchronizing nuclear import of ribosomal proteins with ribosome assembly. Science 338: 666-671. doi:10.1126/science.1226960

Mazumder B, Sampath P, Seshadri V, Maitra RK, DiCorleto PE, Fox PL. 2003. Regulated release of $L 13$ a from the $60 S$ ribosomal subunit as a mechanism of transcript-specific translational control. Cell 115: 187-198. doi:10.1016/S0092-8674(03)00773-6

Melese T, Xue Z. 1995. The nucleolus: an organelle formed by the act of building a ribosome. Curr Opin Cell Biol 7: 319-324. doi:10 .1016/0955-0674(95)80085-9

Melnikov S, Ben-Shem A, Yusupova G, Yusupov M. 2015. Insights into the origin of the nuclear localization signals in conserved ribosomal proteins. Nat Commun 6: 7382. doi:10.1038/ ncomms8382

Milkereit P, Kuhn H, Gas N, Tschochner H. 2003. The pre-ribosomal network. Nucleic Acids Res 31: 799-804. doi:10.1093/nar/gkg165

Moreland RB, Nam HG, Hereford LM, Fried HM. 1985. Identification of a nuclear localization signal of a yeast ribosomal protein. Proc Natl Acad Sci 82: 6561-6565. doi:10.1073/pnas.82.19.6561

Mukhopadhyay R, Ray PS, Arif A, Brady AK, Kinter M, Fox PL. 2008. DAPK-ZIPK-L13a axis constitutes a negative-feedback module regulating inflammatory gene expression. Mol Cell 32: 371-382. doi:10.1016/j.molcel.2008.09.019

Natchiar SK, Myasnikov AG, Kratzat H, Hazemann I, Klaholz BP. 2017. Visualization of chemical modifications in the human 80 ribosome structure. Nature 551: 472-477. doi:10.1038/nature24482

Nguyen Ba AN, Pogoutse A, Provart N, Moses AM. 2009. NLStradamus: a simple Hidden Markov Model for nuclear localiza- tion signal prediction. BMC Bioinformatics 10: 202. doi:10.1186/ 1471-2105-10-202

Ohmayer U, Gil-Hernández A, Sauert M, Martín-Marcos P, Tamame M, Tschochner H, Griesenbeck J, Milkereit P. 2015. Studies on the coordination of ribosomal protein assembly events involved in processing and stabilization of yeast early large ribosomal subunit precursors. PLoS One 10: e0143768. doi:10.1371/journal.pone .0143768

Pelletier J, Thomas G, Volarević S. 2018. Ribosome biogenesis in cancer: new players and therapeutic avenues. Nat Rev Cancer 18: 5163. doi:10.1038/nrc.2017.104

Plafker SM, Macara IG. 2002. Ribosomal protein L12 uses a distinct nuclear import pathway mediated by importin 11. Mol Cell Biol 22: 1266-1275. doi:10.1128/MCB.22.4.1266-1275.2002

Poddar D, Basu A, Baldwin WM III, Kondratov RV, Barik S, Mazumder B. 2013. An extraribosomal function of ribosomal protein L13a in macrophages resolves inflammation. J Immunol 190: 3600-3612. doi:10.4049/jimmunol.1201933

Poddar D, Kaur R, Baldwin WM III, Mazumder B. 2016. L13a-dependent translational control in macrophages limits the pathogenesis of colitis. Cell Mol Immunol 13: 816-827. doi:10.1038/cmi.2015 .53

Rosorius O, Fries B, Stauber RH, Hirschmann N, Bevec D, Hauber J. 2000. Human ribosomal protein L5 contains defined nuclear localization and export signals. J Biol Chem 275: 12061-12068. doi:10 $.1074 / j b c .275 .16 .12061$

Rout MP, Blobel G, Aitchison JD. 1997. A distinct nuclear import pathway used by ribosomal proteins. Cell 89: 715-725. doi:10.1016/ S0092-8674(00)80254-8

Sampath P, Mazumder B, Seshadri V, Fox PL. 2003. Transcript-selective translational silencing by gamma interferon is directed by a novel structural element in the ceruloplasmin mRNA $3^{\prime}$ untranslated region. Mol Cell Biol 23: 1509-1519. doi:10.1128/MCB.23.5 $.1509-1519.2003$

Sampath P, Mazumder B, Seshadri V, Gerber CA, Chavatte L, Kinter M, Ting SM, Dignam JD, Kim S, Driscoll DM, et al. 2004. Noncanonical function of glutamyl-prolyl-tRNA synthetase: gene-specific silencing of translation. Cell 119: 195-208. doi:10 .1016/j.cell.2004.09.030

Tumminia SJ, Hellmann W, Wall JS, Boublik M. 1994. Visualization of protein-nucleic acid interactions involved in the in vitro assembly of the Escherichia coli $50 \mathrm{~S}$ ribosomal subunit. J Mol Biol 235: 1239-1250. doi:10.1006/jmbi.1994.1077

Vyas K, Chaudhuri S, Leaman DW, Komar AA, Musiyenko A, Barik S, Mazumder B. 2009. Genome-wide polysome profiling reveals an inflammation-responsive posttranscriptional operon in gamma interferon-activated monocytes. Mol Cell Biol 29: 458-470. doi:10 .1128/MCB.00824-08

Zhang X, Lai M, Chang W, Yu I, Ding K, Mrazek J, Ng HL, Yang OO, Maslov DA, Zhou ZH. 2016. Structures and stabilization of kinetoplastid-specific split rRNAs revealed by comparing leishmanial and human ribosomes. Nat Commun 7: 13223. doi:10 $.1038 /$ ncomms 13223 

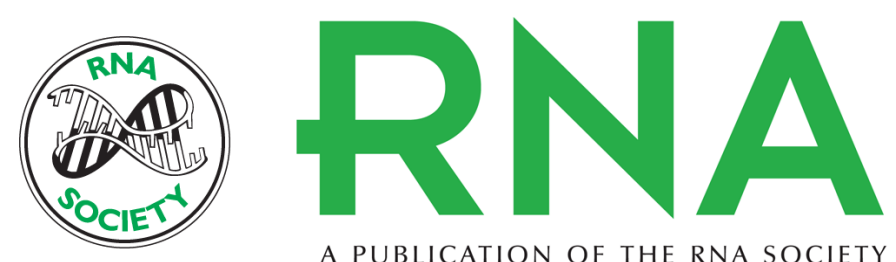

A PUBLICATION OF THE RNA SOCIETY

\section{Mutually exclusive amino acid residues of L13a are responsible for its ribosomal incorporation and translational silencing leading to resolution of inflammation}

Ravinder Kour, Anton A. Komar and Barsanjit Mazumder

RNA 2019 25: 1377-1392 originally published online July 15, 2019

Access the most recent version at doi:10.1261/rna.071118.119

Supplemental Material

References

Creative Commons License

Email Alerting Service
http://rnajournal.cshlp.org/content/suppl/2019/07/15/rna.071118.119.DC1

This article cites 37 articles, 15 of which can be accessed free at: http://rnajournal.cshlp.org/content/25/10/1377.full.html\#ref-list-1

This article is distributed exclusively by the RNA Society for the first 12 months after the full-issue publication date (see http://rnajournal.cshlp.org/site/misc/terms.xhtml). After 12 months, it is available under a Creative Commons License (Attribution-NonCommercial 4.0 International), as described at http://creativecommons.org/licenses/by-nc/4.0/.

Receive free email alerts when new articles cite this article - sign up in the box at the top right corner of the article or click here.

\section{||||||| Providing Precise Solutions for your research.}

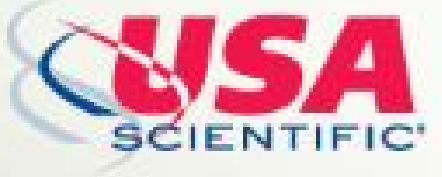

To subscribe to $R N A$ go to:

http://rnajournal.cshlp.org/subscriptions 